\title{
Renormalisation of parabolic stochastic PDEs
}

\author{
The 2oth Takagi Lectures
}

March 9, 2018

\begin{abstract}
M. Hairer
Department of Mathematics, Imperial College London

Email: m.hairer@imperial.ac.uk
\end{abstract}

\begin{abstract}
We give a survey of recent result regarding scaling limits of systems from statistical mechanics, as well as the universality of the behaviour of such systems in so-called cross-over regimes. It transpires that some of these universal objects are described by singular stochastic PDEs. We then give a survey of the recently developed theory of regularity structures which allows to build these objects and to describe some of their properties. We place particular emphasis on the renormalisation procedure required to give meaning to these equations.

These are expanded notes of the $20^{\text {th }}$ Takagi lectures held at Tokyo University on November 4, 2017.
\end{abstract}

\section{Contents}

1 Scaling limits

1.1 The Wiener process . . . . . . . . . . . . . . . . . .

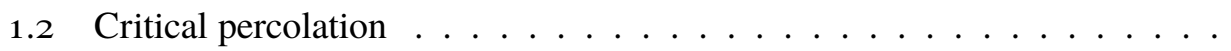

1.3 The Ising model $\ldots \ldots \ldots \ldots \ldots \ldots$

1.4 Interface fluctuations $\ldots \ldots \ldots \ldots \ldots$

1.5 General features and open problem . . . . . . . . . . . . . G

2 Crossover regimes

2.1 The KPZ equation . . . . . . . . . . . . . . . . 11

2.2 The dynamical $\Phi_{3}^{4}$ model $\ldots \ldots \ldots \ldots \ldots$. . . . . . . . . . . . . . . .

3 Singular stochastic PDEs $\quad 15$

$3.1 \quad$ Analysis of the $\Phi^{4}$ model $\ldots \ldots \ldots \ldots \ldots \ldots \ldots$

3.2 Renormalisation . . . . . . . . . . . . . . . . 17

3.3 A simple example . . . . . . . . . . . . . . . . . . . 18 
3.4 Renormalisation of SPDEs . . . . . . . . . . . . . . . . . . . . . . . . . .

3.5 Structure of proof . . . . . . . . . . . . . . . 21

4 Regularity structures 23

4.1 Revisiting the $\Phi^{4}$ equation $\ldots \ldots \ldots \ldots \ldots \ldots$

4.2 Basic definitions . . . . . . . . . . . . . . . 27

4.3 Calculus for regularity structures . . . . . . . . . . 31

4.4 Construction of regularity structures for SPDEs . . . . . . . . . 35

5 Renormalisation 37

5.1 Alternative representation of models . . . . . . . . . . . 38

5.2 Description of the renormalisation procedure ............... 30

5.3 Action on the space of models . . . . . . . . . . . . . . . 40

5.4 Renormalised equations . . . . . . . . . . . . . . . . 43

\section{Scaling limits}

One major achievement of probability theory has been the construction of a number of universal objects that arise naturally as scaling limits of a great number of natural probabilistic models. Let us start by going through a number of examples in order to build some intuition on what scaling limits are, in what context they arise, and what are their properties.

\subsection{The Wiener process}

One of the simplest and most ubiquitous such objects is the Wiener process, which is a random continuous function $W: \mathbf{R}_{+} \rightarrow \mathbf{R}$ with the property that any two nonoverlapping increments are independent and that for any $t>s \geq 0, W(t)-W(s)$ is a centred Gaussian random variable with variance $t-s$. We further impose that $W(0)=0$.

The Wiener process arises naturally as a scaling limit in the following way. Consider an i.i.d. sequence of random variables $\left\{X_{n}\right\}_{n \geq 0}$ that are centred with finite variance $\sigma>0$ and consider the partial sums $S(n)=\sum_{k=0}^{n} X_{k}$ for $n \geq 0$. We extend $S$ to all arguments in $\mathbf{R}_{+}$in a continuous way by linear interpolation:

$$
S(t)=\left(\sum_{k=0}^{\lfloor t\rfloor} X_{k}\right)+(t-\lfloor t\rfloor) X_{\lceil t\rceil} .
$$

We also introduce a family of rescaling operators

$$
\left(\delta_{\lambda}^{(\alpha)} f\right)(t)=\lambda^{-\alpha} f(\lambda t)
$$

The following is then classical [Don51]: 
Theorem 1.1 The family of random functions $S^{\lambda}=\delta_{\lambda}^{(1 / 2)} S$ converges in law on $\mathscr{C}\left(\mathbf{R}_{+}\right)$to a Wiener process as $\lambda \rightarrow \infty$.

Observe that we start from a fixed model (the random function $S$ ) on which we perform a simple rescaling operation to obtain a family of models $S^{\lambda}$ depending on a scale $\lambda$. This scale is then sent to infinity in order to obtain a scaling limit. It follows immediately that $W$ itself is invariant under the action of the rescaling $\delta_{\lambda}^{(1 / 2)}$ since

$$
\delta_{\lambda}^{(1 / 2)} W=\lim _{\mu \rightarrow \infty} \delta_{\lambda}^{(1 / 2)} \delta_{\mu}^{(1 / 2)} S=\lim _{\mu \rightarrow \infty} \delta_{\lambda+\mu}^{(1 / 2)} S=W
$$

where all limits and identities are in law. The exponent $1 / 2$ appearing in this expression is called the scaling exponent of $W$. Another important feature of $W$ is that it exhibits a form of stationarity, or translation invariance. Indeed, the partial sums $S(n)$ are such that, for any fixed $k \geq 0$, the random sequence $\tilde{S}(n)=$ $S(k+n)-S(k)$ is equal in law to the random sequence $S$ itself. It immediately follows that $W$ satisfies the analogous property, namely that $W(\cdot+s)-W(s)$ is equal in law to $W$ itself, for every $s \geq 0$. A final and equally crucial property enjoyed by the Wiener process $W$ is the Markov property: for any time interval $I \subset \mathbf{R}_{+}$, the law of $W\left\lceil I\right.$, conditional on $W\left\lceil I^{c}\right.$, only depends on the values of $W$ on the boundary of $I$. This is again a simple consequence of the fact that $S$ satisfies a similar property, but with fattened boundaries. More precisely, for $[a, b] \subset \mathbf{R}_{+}$, the law of $S \uparrow[a, b]$, conditional on $S \uparrow[a, b]^{c}$, only depends on the values of $S$ on $[a-1, a] \cup[b, b+1]$.

\subsection{Critical percolation}

Scaling limits are of course not restricted to functions and, as a matter of fact, several major recent results concern scaling limits of more sophisticated objects. Let us briefly describe two of these results, namely critical percolation and the construction of the "Brownian plane". In the first example, our starting point is the infinite regular triangular lattice, together with an assignment of a random variable $\omega_{x} \in\{0,1\}$ for every vertex $x$ of the lattice. We assume that the $\omega_{x}$ are i.i.d. Bernoulli random variables with parameter $p$.

There are two different ways in which we can take a scaling limit in this case. The first "naïve" one, similarly to (1.1), is to extend $\omega$ to the whole plane by some local interpolation procedure that respects the symmetries of the lattice. (Local in the sense that the value at a given point $x$ only depends on the values of $\omega_{y}$ for those vertices $y$ that intersect a neighbourhood of $x$ of some fixed radius.) This yields a random function $\omega: \mathbf{R}^{2} \rightarrow \mathbf{R}$ and we can ask whether this random function admits a scaling limit. A variant of Theorem 1.1 shows that this is indeed the case, and 
we have the convergence in law

$$
\lim _{\lambda \rightarrow \infty} \delta_{\lambda}^{(-1)}(\omega-p)=\xi
$$

where $\xi$ is a "white noise". This time, the limit is not a random function, but a random Schwartz distribution: for any collection of test functions $\varphi_{k}$, the random variables $\xi\left(\varphi_{k}\right)$ are jointly centred Gaussian and one has

$$
\mathbf{E} \xi(\varphi) \xi(\psi)=\langle\varphi, \psi\rangle,
$$

where the scalar product is taken in $L^{2}$. In particular, if $\delta_{\varepsilon}$ is an $\varepsilon$-approximation of a Dirac mass, one has $\mathbf{E} \xi\left(\delta_{\varepsilon}\right)^{2} \sim \varepsilon^{-2}$, which shows that there is no hope to be able to find a continuous version of $\xi$. The fact that white noise can only be realised as a random distribution is directly linked to the fact that it arises as a scaling limit (1.2) with a negative exponent.

However, while this white noise scaling limit does describe the large-scale behaviour of local averages of the random variables $\omega_{x}$, it does not tell us anything about the large-scale behaviour of many interesting observables that are naturally built from $\omega$. In particular, one would like to be able to give a description of the large-scale geometry of the subset $\left\{x: w_{x}=1\right\}$ of the triangular lattice, together with the connectivity structure induced by that of the underlying lattice. One possible way of encoding this connectivity structure is by viewing it as a collection of "quads" (i.e. homeomorphisms $Q:[0,1]^{2} \rightarrow \mathbf{R}^{2}$ ), where we keep precisely those quads $Q$ such that there exists a percolation cluster intersecting the two opposite edges $Q(\{0\} \times[0,1])$ and $Q(\{1\} \times[0,1])$. (We can view the percolation clusters as closed subsets of the plane by for example linking neighbouring open sites with a line segment. The precise way in which this is done is irrelevant for what follows.) Writing $Q$ for the space of all quads, which can be endowed with a natural distance function, a percolation configuration is therefore encoded by a random subset $S_{\omega} \subset \mathbb{Q}$ satisfying furthermore a number of natural consistency and monotonicity properties. The space $\mathcal{X}$ of all such subsets can itself be endowed with a natural topology. The is furthermore a natural scaling operation $\delta_{\lambda}: \mathbb{Q} \rightarrow \mathbb{Q}$ on the space of quads by setting

$$
\left(\mathcal{S}_{\lambda} Q\right)(x)=\lambda Q(x) .
$$

This in yields a map $\delta_{\lambda}: X \rightarrow X$ on subsets of $\mathbb{Q}$ which preserves the consistence and monotonicity properties alluded to above. It was then shown by Schramm and Smirnov [SS11] that if one sets $p=p_{c}=\frac{1}{2}$ (the critical value for percolation on the triangular lattice [Kes80]), then the laws of the sequence of random sets $\delta_{\lambda} S_{\omega}$ is tight in $\mathcal{X}$ as $\lambda \rightarrow \infty$ and its accumulation points are non-degenerate. The limiting $\mathcal{X}$-valued random variable $\tilde{S}$ (which is conjectured to be unique) has again natural 
scale invariance, translation invariance, and Markov properties. In this particular case, it is furthermore rotation invariant and actually conformal invariant.

It is interesting to note that the information encoded in the scaling limit $\xi$ and that encoded in the scaling limit $\tilde{S}$ are completely different, although both $\delta_{\lambda}^{(-1)} w$ and $\delta_{\lambda} S_{\omega}$ encode the exact same information for any fixed $\lambda>0$. What "completely different" means in this context is that the noise (in the sense of Tsirelson [Tsio4]) generated by $\xi$ is "white" while that generated by $\tilde{S}$ is "black" [SS11].

\subsection{The Ising model}

Another example of scaling limit was recently obtained by [CGN15, CGN16] for the $2 \mathrm{D}$ Ising model at criticality. Recall that the Ising model in a domain $\Lambda \subset \mathbf{Z}^{2}$ is given by the measure on $\{ \pm 1\}^{\Lambda}$ assigning to each configuration $\sigma$ a probability proportional to $\exp (-\beta H(\sigma))$, where the Hamiltonian is given by

$$
H(\sigma)=-\sum_{x \sim y} \sigma_{x} \sigma_{y}
$$

Here, $x \sim y$ if and only if $x$ and $y$ are nearest neighbours in $\mathbf{Z}^{2}$ and the values of $\sigma$ outside of $\Lambda$ are considered to be fixed. It is well-known that the Ising model exhibits a phase transition: there exists a value $\beta_{c}>0$ such that, for $\beta \leq \beta_{c}$, there exists a unique probability measure on $\{ \pm 1\}^{\mathbf{Z}^{2}}$ with the property that, for every $\Lambda \subset \mathbf{Z}^{2}$ finite, the conditional measure on $\{ \pm 1\}^{\Lambda}$ is as above. For $\beta>\beta_{c}$ however, while such measure do still exist, they are not unique anymore. In fact, there are two such measures, one in which a majority of spins take the value +1 , and one in which a majority of spins take the value -1 . At criticality (i.e. for $\beta=\beta_{c}$ ), local averages of spins exhibit a non-trivial scaling limit in the sense that if we extend $\sigma$ to all of $\mathbf{R}^{2}$ in a way similar to above, then $\delta_{\lambda}^{(-1 / 8)} \sigma$ converges in law to a non-trivial measure $\mathbf{P}_{c}$ on the space of distributions on $\mathbf{R}^{2}$. Unlike in the previous examples of this type, the measure $\mathbf{P}_{c}$ is not Gaussian, in fact its tails are lighter than Gaussian. Again, the measure $\mathbf{P}_{c}$ is scaling invariant (with exponent $-1 / 8$ ), translation invariant, and satisfies the spatial Markov property.

\subsection{Interface fluctuations}

Our final example of scaling limit is the closest one to the type of problems considered in these notes, and this is also the only example of scaling limit in which one considers models that depend on both space and time. Consider a model of one-dimensional interface growth where the interface is modelled as the graph of a function $h: \mathbf{Z} \rightarrow \mathbf{Z}$. We furthermore restrict the state space to those functions $h$ such that $h(x+1)-h(x) \in\{ \pm 1\}$ for every $x \in \mathbf{Z}$. A simple dynamic on such functions is given by the following. To each site $x \in \mathbf{Z}$, we then assign a 
Poisson process $t \mapsto N_{x}(t)$, with all the $N_{x}$ 's being i.i.d. and having the same rate (say 1). This allows us to build a dynamic on the space of height functions $h$ in the following way. Whenever one of the processes $N_{x}$ jumps, we update $h$ in the following way. If $h_{t}$ has a local minimum at $x$, then we set $h_{t^{+}}(x)=h_{t}(x)+2$. If $h_{t}$ has a local maximum at $x$, then we set $h_{t^{+}}(x)=h_{t}(x)-2$, but only with some fixed probability $q \in[0,1]$, independently of the processes $N_{x}$. If $h_{t}$ has neither a local minimum, nor a local maximum at $x$, then we leave it unchanged. Note that the case $q=1$ is special since in this case $h$ and $-h$ have the same distribution.

It is possible to show [Lig85] that this dynamic is indeed well-defined (this is not completely obvious since infinitely many of the processes $N_{x}$ perform a jump in any given time interval). Depending on the literature, this process is referred to as the height function for the asymmetric simple exclusion process (symmetric in the case $q=1$ ) or as the SOS (Solid On Solid) model. This process has the property that, for any given value of $q$, the simple random walk is an invariant measure for it. This suggests that, in order to obtain a scaling limit, the initial condition $h_{0}$ should be chosen such that $\delta_{\lambda}^{(1 / 2)} h_{0}$ converges in law to some limit $\tilde{h}_{0}$. In this situation, since time and space play different roles, there is no reason a priori to consider time scales of the same order as the spatial scale, so we consider scaling operators with two scaling exponents like so:

$$
\left(\delta_{\lambda}^{(\alpha, \beta)} h\right)(x, t)=\lambda^{\alpha} h\left(\lambda x, \lambda^{\beta} t\right) .
$$

In the symmetric case $q=1$, one then has the following result [DMIPP84, Rav92]:

Theorem 1.2 For $q=1$, let $h_{0}^{\lambda}$ be a sequence of initial conditions for the symmetric SOS model such that $\delta_{\lambda}^{(1 / 2)} h_{0}^{\lambda}$ converges to a limit $h_{0}$ in $\mathscr{C}_{b}(\mathbf{R})$. Then, the process $\delta_{\lambda}^{(1 / 2,2)} h$ converges in law as $\lambda \rightarrow \infty$ to the solution to the stochastic heat equation with initial condition $h_{0}$ :

$$
\partial_{t} h=\partial_{x}^{2} h+\xi
$$

where $\xi$ denotes space-time white noise.

Note again that the process $h$ satisfies the (space-time) Markov property as a consequence of the fact that it has a purely local specification. In the case of interface fluctuation models, it turns out that symmetric models behave very differently at large scales from asymmetric ones. Indeed, the following has very recently been shown in [MQR17].

Theorem 1.3 For $q=0$, let $h_{0}^{\lambda}$ be a sequence of initial conditions for the totally asymmetric SOS model such that $\delta_{\lambda}^{(1 / 2)} h_{0}^{\lambda}$ converges to a limit $h_{0}$ in $\mathscr{C}_{b}(\mathbf{R})$. Then, the process $\delta_{\lambda}^{(1 / 2,3 / 2)} h$ converges in law as $\lambda \rightarrow \infty$ to a limiting non-trivial Markov process $h$. 
Note that the time-scaling exponent appearing here is different from the one appearing in the symmetric case. Furthermore, [MQR17] give a full characterisation of the transition probabilities of the limiting process $h$, which shows in particular that it is not Gaussian. Unlike in the symmetric case, the scaling limit in the totally asymmetric case (also called the "KPZ fixed point") is not described as the solution to a stochastic PDE. It does however have a characterisation via a type of noisy Lax-Oleinik formula [CQR15], although this characterisation is not complete. (There are only tightness results and partial characterisations of the "Airy sheet" which acts as a driving noise.)

\subsection{General features and open problem}

Despite the impression the reader may obtain from these various examples, these scaling limits are rigorously much less understood than one would think. For example, the Ising model admits two very natural dynamics preserving its Gibbs measure: the Glauber dynamic in which one randomly flips spins and then rejects some moves in order to enforce the correct invariant measure and the Kawasaki dynamic, where the elementary moves are given by exchanging neighbouring spins. It is then natural to ask whether there is a scaling limit for the process $(t, x) \mapsto \sigma_{x}(t)$ induced by these dynamics at the critical parameter $\beta_{c}$. It is conjectured that this is indeed the case, but one does not even have a conjecture for the correct value of the corresponding dynamical scaling exponents! Similarly, one does not know what the correct scaling $\alpha$ is which guarantees that $\delta_{\lambda}^{(-\alpha)} \sigma$ converges to a non-trivial limit for $\sigma$ given by the $3^{\mathrm{D}}$ Ising model at criticality, although recent breakthrough results [ESPP $\left.{ }^{+} 12, \mathrm{ESPP}^{+} 14\right]$ yield a constructive, albeit nonrigorous, way of approximating this exponent (the best known approximation to date is about 0.51815 which does not appear to be a rational number with small denominator).

Another feature of these scaling limits that still lacks rigorous mathematical understanding is their universality. In all of the examples given above, we have mathematical theorems exhibiting scaling limits for one specific model, typically one that is exactly solvable. It is (loosely) conjectured that in all cases, the same scaling limit arises when starting from any model that exhibits the same broad features as the specific model we consider. This is only well understood in the first example of the Wiener process, where the functional central limit theorem was shown to hold for a very large class of models.

In the case of critical percolation for example, one expects to obtain the same scaling limit for its connectivity structure, independently of the precise structure of the underlying graph, provided that it is planar and sufficiently regular. In the case of the Ising model, one also expects the same results for a large class of underlying grids, similarly to the case of percolation. (For a different class of observables, this was indeed shown in [CS12], although the class of grids considered there is 
still rather rigid and interactions are still restricted to nearest-neighbours in order to enforce a suitable type of integrability.) However, when considering the scaling limit of the magnetisation as described above, one also expects many continuous models to fall into the same universality class. For example, the $\Phi_{2}^{4}$ measure constructed in [Nel66, GJ87, BFS83] is a measure on distributions on $\mathbf{R}^{2}$ which can formally be thought of as having density proportional to

$$
\exp \left(-\int_{\mathbf{R}^{2}}\left(|\nabla \Phi(x)|^{2}+\Phi^{4}(x)-C \Phi^{2}(x)\right) d x\right)
$$

with respect to "Lebesgue measure" (which of course does not exist). This models the same situation as that of the Ising model and depends on a parameter $C$ which plays a role similar to that of the inverse temperature $\beta$. It is natural to conjecture that, when this parameter is set equal to its critical value, the large-scale behaviour of the $\Phi_{2}^{4}$ measure is precisely the same as that of the Ising model.

Finally, in the case of interface fluctuations, one expects the KPZ fixed point to be the scaling limit of a very large class of asymmetric models. In particular, one expects it to arise as the scaling limit of the SOS model for any $q \in[0,1)$, but also as the scaling limit of various other models like the Eden model, the ballistic deposition model, etc. The only rigorous results in this direction were obtained for models exhibiting a type of "integrable structure", for which it is possible to show that certain observables converge to the corresponding observables of the KPZ fixed point. See for example [Johoo, TWo8, BC14] for some results in this direction.

All this suggests that answering the following open problem would allow to make substantial progress.

Open Problem 1 For any $d \geq 2$, characterise all pairs $(\alpha, \eta)$ such that $\alpha \in \mathbf{R}$ and $\eta$ is a random distribution on $\mathbf{R}^{d}$ which is stationary, satisfies $\delta_{\lambda}^{(-\alpha)} \eta=\eta$ in law, and satisfies the spatial Markov property.

Remark 1.4 If $\alpha \geq 0$, one cannot in general expect $\eta$ to be both self-similar with exponent $\alpha$ and stationary. However, at least for non-integer values, one can still expect stationarity in a generalised sense where one recenters $\eta$ in such a way that its value and the relevant number of derivatives vanish at the origin. This is precisely what happens in the case of the two-sided Wiener process for example which is invariant under the action of the group of translations given by

$$
\left(\tau_{s} W\right)(t)=W(t+s)-W(s)
$$

Remark 1.5 There are many natural variants of this question. For example, it would be natural to also impose rotation invariance. It would also be natural to add 
time as an additional dimension and to allow the scaling exponent to be different for time scalings. Finally, it would be natural to impose restrictions on the tail behaviour of $\eta$, for example that $\eta(\varphi)$ has moments of all orders for every $\varphi \in \mathscr{C}_{0}^{\infty}$. (In the case $d=1$, this property is known to significantly reduce the possible candidates.)

\section{Crossover regimes}

We have seen in the previous section that there is a great interest in finding and characterising scaling limits, as well as the corresponding universality classes. (The universality class for a given scaling limit $\eta$ consists of the collection of all those models that converge to $\eta$ under a suitable rescaling operation which leaves $\eta$ invariant.) Unfortunately, when considering scaling limits of "random field" type, the only situation that is mathematically well-understood in the sense that we have a mathematically rigorous understanding of both the scaling limit itself and of its universality class is the Gaussian case. There are some non-Gaussian cases that can be characterised by explicit formulae, which include the case of twodimensional models (without time-dependence), as well as the KPZ fixed point. In these cases however, universality statements are restricted to microscopic models that share some of the integrability properties of their scaling limits.

This suggests that one possible way of improving our understanding of the largescale behaviour of random systems is to consider what happens "in the vicinity" of systems that rescale to a Gaussian scaling limit. More precisely, consider a family $\left\{S^{(\varepsilon)}\right\}_{\varepsilon \in D}$ of random functions on $\mathbf{R}^{d}$ depending continuously on $\varepsilon$, where $D \subset \mathbf{R}^{m}$ is some closed set containing the origin (think of $D=[0,1]$ ). Assume that this family is such that, for some scaling exponent $\alpha, \delta_{\lambda}^{(\alpha)} S^{(0)}$ converges, as $\lambda \rightarrow \infty$, to a Gaussian scaling limit $\eta$. One can then find situations in which this is no longer the case for $\varepsilon \neq 0$, so that one expects to have some different scaling exponent $\beta \neq \alpha$ (or pair of space-time scaling exponents) such that, for fixed $\varepsilon \neq 0, \delta_{\lambda}^{(\beta)} S^{(\varepsilon)}$ converges, as $\lambda \rightarrow \infty$, to some non-Gaussian scaling limit. (It is usually completely out of reach of current mathematical technology to prove such a thing. In general, it may also happen that $D$ is further broken into subregions on which $\beta$ takes different values.) It is then natural to ask whether it is possible to prove a non-trivial limiting result for limits of the form $\delta_{\lambda_{\varepsilon}}^{(\alpha)} S^{(\varepsilon)}$ as $\varepsilon \rightarrow 0$ with $\lim _{\varepsilon \rightarrow 0} \lambda_{\varepsilon}=\infty$.

By a simple diagonal argument, using the continuity of $\varepsilon \mapsto S^{(\varepsilon)}$, if $\lambda_{\varepsilon}$ diverges sufficiently slowly, one still has $\lim _{\varepsilon \rightarrow 0} \delta_{\lambda_{\varepsilon}}^{(\alpha)} S^{(\varepsilon)}=\eta$. On the other hand, since $\beta \neq \alpha$, one expects $\delta_{\lambda_{\varepsilon}}^{(\alpha)} S^{(\varepsilon)}$ to either converge to 0 or blow up as $\varepsilon \rightarrow 0$ for $\lambda_{\varepsilon}$ diverging sufficiently fast. This suggests the existence of a non-trivial intermediate regime, called the crossover regime for which some non-trivial limits different from 
$\eta$ are obtained.

In these notes, we'll argue that in many cases of interest, the behaviour one observes is the following. There exists a family $\eta^{v}$ of possible limits, parametrised by $v$ belonging to some closed subset $K \subset \mathbf{R}^{m}$ containing the origin for some $m \geq 0$, with $\eta^{0}=\eta$, the original Gaussian scaling limit. Each element of the family is typically stationary (possibly in a generalised sense as already described earlier) and Markovian, but it is this time only the family as a whole which is scaling invariant and not individual members of the family. More precisely, there exists an action $T$ of $\left(\mathbf{R}_{+}, \cdot\right)$ onto $K$ such that

$$
\delta_{\lambda}^{(\alpha)} \eta^{v}=\eta^{T^{\lambda} v}
$$

and such that $\lim _{\lambda \rightarrow 0} T^{\lambda} v=0$ for every $v \in K$. In other words, the family is invariant under the scaling operations with exponent $\alpha$ and, at small scales, every member of the family looks like $\eta$. Furthermore, the family $\left\{\eta^{v}\right\}_{v \in K}$ is universal in the sense that, for a very large class of models $\left\{S^{(\varepsilon)}\right\}_{\varepsilon \in D}$ as above, one can find a map $\varepsilon \mapsto\left(\lambda_{\varepsilon}, v_{\varepsilon}\right)$ such that

1. $v_{\varepsilon}$ is uniformly bounded on $\varepsilon \in D \backslash\{0\}$,

2. there are sequences $\varepsilon_{n} \rightarrow 0$ such that $v_{\varepsilon_{n}}$ remains uniformly bounded away from 0 ,

3. $\lambda_{\varepsilon} \rightarrow \infty$ as $\varepsilon \rightarrow 0$,

4. one has $\delta_{\lambda_{\varepsilon}}^{(\alpha)} S^{(\varepsilon)} \rightarrow \eta^{v_{\varepsilon}}$ as $\varepsilon \rightarrow 0$.

Note that there is necessarily some indeterminacy here since, if $\varepsilon \mapsto\left(\lambda_{\varepsilon}, v_{\varepsilon}\right)$ is one map satisfying the above properties then it follows from (2.1) that, for any fixed $\mu>0$, the map $\varepsilon \mapsto\left(\mu \lambda_{\varepsilon}, T^{\mu} v_{\varepsilon}\right)$ also satisfies the same properties.

Remark 2.1 In all the examples below, the underlying space $\mathbf{R}^{d}$ on which our random distributions are defined naturally breaks into a time component and $d-1$ spatial components. In this case, scalings are typically different for these two types of components, but the discussion above remains unchanged otherwise.

Remark 2.2 There are some situations where major technical difficulties arise due to the fact that space is unbounded. Although this is very much case-dependent, there are often natural ways of defining the models $S^{(\varepsilon)}$ and $\eta^{v}$ on a finite domain rather than the whole space. In this case, the same discussion usually applies but with two caveats. First, there may be additional parameters governing boundary conditions and matching these between $S^{(\varepsilon)}$ and $\eta^{v}$ may be non-trivial, see [CS16, GH18]. Second, if we normalise the $\eta^{v}$ to be defined on a domain of size 1 , then the models $S^{(\varepsilon)}$ have to be defined on a domain of size $\lambda_{\varepsilon}$ for the third property above to hold.

We now discuss a couple of concrete examples to illustrate the situation. 


\subsection{The KPZ equation}

Let us now show how the KPZ equation [KPZ86] fits into the framework described above. Recall that the KPZ equation is the stochastic partial differential equation formally given by

$$
\partial_{t} h=\partial_{x}^{2} h+\left(\partial_{x} h\right)^{2}+\xi
$$

where $\xi$ denotes space-time white noise. The reason why this expression is only formal is that solutions are not differentiable, so the expression $\left(\partial_{x} h\right)^{2}$ has no classical meaning. It is however possible to give an unambiguous notion of "solution" to this equation, the so-called Hopf-Cole solution, see [BG97, QS15]. As a matter of fact, this gives a meaning the family of equations formally given by

$$
\partial_{t} h=\partial_{x}^{2} h+c_{1}\left(\partial_{x} h\right)^{2}+c_{2}+\xi,
$$

and we write $\eta^{c}$ with $c=\left(c_{1}, c_{2}\right) \in \mathbf{R}^{2}$ for its solutions.

Remark 2.3 There is a rather non-trivial question arising here which is that of the choice of initial condition. It so happens that it is possible to construct solutions that are stationary in space-time, modulo recentering, and these are the solutions that we consider. There is a subtlety here in that these solutions are themselves not unique since Brownian motion with drift is invariant for the corresponding Markov process for any value of the drift, see [FQ15]. We lift this ambiguity by choosing the (unique) solution with zero drift.

Using the scale invariance properties of white noise, it can be verified that, at least at a formal level, the family of solutions to (2.2) does satisfy (2.1) with

$$
T^{\lambda}\left(c_{1}, c_{2}\right)=\left(\lambda^{1 / 2} c_{1}, \lambda^{3 / 2} c_{2}\right) .
$$

It happens that the Hopf-Cole solutions are such that this identity does indeed hold for them as well.

Remark 2.4 We could of course also add constants in front of the terms $\partial_{x}^{2} h$ and $\xi$, but it can be shown that it is always possible to rescale solutions in such a way that these constants are equal to 1 . As a matter of fact, even the constants $c_{1}$ and $c_{2}$ can be eliminated by performing first a rescaling to set $c_{1}$ to 1 by (2.3) and then perform a height shift, i.e. considering transformations of the type $h(t, x) \mapsto h(t, x)-c t$, which allow to set $c_{2}$ to 0 without loss of generality. This is why we usually talk about "the" KPZ equation, rather than the family of KPZ equations.

\subsubsection{Convergence of the SOS model}

Let now $S^{(\varepsilon)}$ denote the stationary SOS model with $q=1-\sqrt{\varepsilon}$ and with fixed time distribution given by that of the simple random walk. We furthermore perform a 
height shift in order to set the average speed of the interface to zero, so that if $h^{(\varepsilon)}$ denotes the stationary SOS model with $q=1-\sqrt{\varepsilon}$ as defined in Section 1.4, we set

$$
S^{(\varepsilon)}(t, x)=h_{t}^{(\varepsilon)}(x)-\sqrt{\varepsilon} t .
$$

It was then shown in [BG97] that if we choose $\lambda_{\varepsilon}=1 / \varepsilon$, and consider the processes $h^{(\varepsilon)}=\delta_{\lambda_{\varepsilon}}^{(1 / 2,2)} S^{(\varepsilon)}$, rescaled as in the symmetric case mentioned in Section 1.4, then $h^{(\varepsilon)}$ does converge to the Hopf-Cole solution to the KPZ equation.

This can indeed be cast into the framework exposed at the beginning of this section: We have $D=[0,1]$ and $K=\mathbf{R}^{2}$, and the mapping $\varepsilon \mapsto\left(\lambda_{\varepsilon}, v_{\varepsilon}\right)$ is simply given by $\varepsilon \mapsto\left(\varepsilon^{-1}, c\right)$, where $c$ denotes the specific value of the constants in the $\mathrm{KPZ}$ equation appearing in the result of [BG97]. In this case, this seems very much overkill. The interesting fact however is that the limiting object obtained in the crossover regime, namely the solution to the KPZ equation, is itself universal in the sense that it arise in many more situations.

\subsubsection{A universality result}

Consider for example a Gaussian random field $\zeta: \mathbf{R}^{2} \rightarrow \mathbf{R}$ with a correlation function $\varrho$ that is compactly supported and such that $\int \varrho=1$. Then, a simple calculation shows that $\delta_{\lambda}^{(-3 / 2,2)} \zeta$ converges to space-time white noise as $\lambda \rightarrow \infty$. In particular, if we denote by $h^{(0)}$ the solution to

$$
\partial_{t} h^{(0)}=\partial_{x}^{2} h^{(0)}+\zeta
$$

then $S_{\lambda}^{(1 / 2,2)} h^{(0)}$ converges in law as $\lambda \rightarrow \infty$ to the solution to the stochastic heat equation (1.3). Consider now perturbations of (2.4) of the type

$$
\partial_{t} h=\partial_{x}^{2} h+P\left(\partial_{x} h\right)+\zeta,
$$

where $P$ is an even polynomial of some fixed degree $2 \mathrm{~m}$. The rationale for restricting ourselves to even polynomials is that it is natural for interface growth models to be symmetric under the exchange $x \leftrightarrow-x$. Furthermore, the rationale for considering perturbations depending only on $\partial_{x} h$ and not on $h$ itself is that the environment in which our interface moves is homogeneous.

This again fits the framework described above, but this time it is the polynomial $P$ itself which plays the role of the parameter $\varepsilon$, so that one has $D \approx \mathbf{R}^{m+1}$, with the identification between polynomials and $\mathbf{R}^{m+1}$ given by

$$
P=P_{\varepsilon}, \quad P_{\varepsilon}(u)=\sum_{p=0}^{m} \varepsilon_{p} u^{2 p} .
$$


What behaviour does one expect for the large-scale behaviour of (2.5) for small $P$ ? Note that while (2.4) only admits a stationary solution in a generalised sense, its spatial derivative $\partial_{x} h^{(0)}$ admits a genuine stationary solution. Since it is Gaussian, this means that there exists a centred Gaussian measure $\nu_{0}$ such that $\partial_{x} h^{(0)}(t, x)$ has law $\nu$ for any fixed $(t, x)$.

It is then not unreasonable to expect that the effective behaviour of $P\left(\partial_{x} h\right)$ in (2.5) is close to that of the constant $\int P(u) \nu(d u)$. Indeed, it is not difficult to show that this is the case in the sense that if we choose for example

$$
\lambda_{\varepsilon}=\left|P_{\varepsilon}\right|^{-2 / 3}
$$

where $\left|P_{\varepsilon}\right|=\sum_{p}\left|\varepsilon_{p}\right|$, then $\delta_{\lambda_{\varepsilon}}^{(1 / 2,2)} h^{\left(P_{\varepsilon}\right)}$ is uniformly close for $\left|P_{\varepsilon}\right|$ small to the solution to

$$
\partial_{t} h=\partial_{x}^{2} h+c_{\varepsilon}+\xi, \quad c_{\varepsilon} \stackrel{\text { def }}{=} \lambda_{\varepsilon}^{3 / 2} \int P(u) \nu(d u) .
$$

Here, we wrote $h^{(P)}$ for the solution to (2.5). Since (2.6) guarantees that $c_{\varepsilon}$ is uniformly bounded, this is indeed a result of the type described above, but it is somewhat disappointing. Indeed, as already mentioned, the solution to (2.7) differs from that of (1.3) by a simple shift, so that we really haven't obtained a different object at all.

Instead, we should focus on what happens when $\int P d \nu$ vanishes, in which case $c_{\varepsilon}$ vanishes, so that we have a chance of being able to look at larger scales. It turns out that a more interesting choice of scale $\lambda_{\varepsilon}$ which leads to non-trivial behaviour is given by

$$
\lambda_{\varepsilon}^{-1}=\left|P_{\varepsilon}\right|^{2}+\left|\int P(u) \nu(d u)\right|^{2 / 3} .
$$

We see that if $\int P d \nu$ is of the same order as $\left|P_{\varepsilon}\right|$, then this scale is of the same order as that in (2.6). If however $\int P d \nu$ is much smaller, then this scale is much larger than the previous one.

Remark 2.5 The precise form of $\lambda_{\varepsilon}$ does not matter as long as it satisfies the bounds $\lambda_{\varepsilon} \lesssim\left|P_{\varepsilon}\right|^{-2}$ and $\lambda_{\varepsilon} \lesssim\left|\int P d \nu\right|^{-2 / 3}$.

The main result of [ $\left[\overline{\mathrm{HQ}_{15}}\right]$ can then be loosely formulated as follows.

Theorem 2.6 There exists a trilinear form $F: P \mapsto F(P, P, P)$ such that, with the choice (2.8), $\delta_{\lambda_{\varepsilon}}^{(1 / 2,2)} h^{\left(P_{\varepsilon}\right)}$ is uniformly close for $\left|P_{\varepsilon}\right|$ small to the Hopf-Cole solution to (2.2) with

$$
c_{1}=\frac{\lambda_{\varepsilon}^{1 / 2}}{2} \int P^{\prime \prime}(u) \nu(d u), \quad c_{2}=\lambda_{\varepsilon}^{3 / 2}\left(\int P(u) \nu(d u)+F(P, P, P)\right) .
$$


Similar results have recently been obtained in [GP16], where the authors also allow for non-polynomial $P$, but with the caveat that they are restricted to a slightly different class of models for which the invariant measure is explicitly known. The work [ $\mathrm{HX} 18 \mathrm{~b}]$ also extends the above results to general $P$, this time for the same model as (2.5).

Remark 2.7 The formulation of Theorem 2.6 is not complete as it leaves out the question of the initial condition. Furthermore, the results just mentioned only hold for (2.5) defined on a torus of size $\lambda_{\varepsilon}$ instead of the whole real line. The reason is that, on the whole real line, it is not even clear for which initial conditions (2.5) admits local solutions!

These results suggest the following conjecture which would be a very strong universality statement for the KPZ equation.

Open Problem 2 Show that the solution to the KPZ equation is the unique continuous stationary (in the generalised sense) space-time Markov process $h$ invariant under $x \leftrightarrow-x$ which has the property that $S_{\lambda}^{(1 / 2,2)} h$ converges to the solution to the stochastic heat equation as $\lambda \rightarrow 0$ and $\delta_{\lambda}^{(1 / 2,3 / 2)} h$ converges to the KPZ fixed point as $\lambda \rightarrow \infty$.

\subsection{The dynamical $\Phi_{3}^{4}$ model}

This is a family of models which should formally be thought of as the solutions to the stochastic PDEs

$$
\partial_{t} \Phi=\Delta \Phi+c_{1} \Phi-c_{2} \Phi^{3}+\xi,
$$

where $\xi$ again denotes space-time white noise, but the spatial variable now takes values in $\mathbf{R}^{3}$. This time, the model with $c=\left(c_{1}, c_{2}\right)=0$ is invariant under the action of the scaling operators $\delta_{\lambda_{\varepsilon}}^{(-1 / 2,2)}$. A formal calculation suggests that this family of models satisfies (2.1) with the action $T^{\lambda}$ given by $T^{\lambda} c=\left(\lambda^{3 / 2} c_{1}, \lambda^{1 / 2} c_{2}\right)$. This is incorrect however, instead it is the case that it does satisfy (2.1), but with the modified action

$$
T^{\lambda} c=\left(\lambda^{3 / 2}\left(c_{1}+a c_{2}^{3} \log \lambda\right), \lambda^{1 / 2} c_{2}\right),
$$

where $a$ is some fixed explicitly computable constant. (One readily verifies that this action satisfies the semigroup property $T^{\lambda} T^{\mu}=T^{\lambda \mu}$.)

This time, while the constant $c_{2}$ can always be set to 1 by a simple rescaling operation, there is no way in general to adjust the value of $c_{1}$ by a simple local transformation, so we do genuinely have a one-parameter family of distinct models indexed by $c_{2}$. This parameter plays a role similar to that of the temperature in the Ising model, and the two models are expected to fall into the same universality 
class in the sense that one expects there to exist a critical value for $c_{2}$ at which (2.9) admits a scaling limit which coincides with the scaling limit for the Glauber dynamic of the critical $3 \mathrm{D}$ Ising model. Let us reiterate again that although this is an accepted fact in the physics literature, from a mathematical perspective it is highly speculative. In particular, neither of these scaling limits is known to exist, and there does not even exist a precise conjecture for the corresponding scaling exponents.

It was shown in [HX18a] that, if one considers models of the type

$$
\partial_{t} \Phi=\Delta \Phi-V^{\prime}(\Phi)+\zeta,
$$

for even polynomials $V: \mathbf{R} \rightarrow \mathbf{R}$ of fixed degree and $\zeta$ a Gaussian random field with compactly supported correlations as above, then a result analogous to (2.6) holds (but with slightly different expressions for $c$ and the scale $\lambda$ ). It was also shown in [MW17] that the two-dimensional analogue of this model arises in the crossover regime for the Glauber dynamic of Ising-Kac models, namely spin models with Hamiltonian

$$
H_{\varepsilon}(\sigma)=-\varepsilon^{2} \sum_{x, y} \sigma_{x} \sigma_{y} K(\varepsilon(x-y)) .
$$

A similar result is expected to hold in the three-dimensional case as well.

\section{Singular stochastic PDEs}

In the previous section, we have seen singular stochastic PDEs appearing as universal objects describing the crossover regimes for various models from statistical mechanics. These equations were singular in the sense that they involved nonlinearities that appear to have no canonical meaning since they involve products of distributions of negative order. For this reason, it is not clear how processes like the solutions to (2.22.9) should be defined and, a fortiori, how universality results like the ones presented above should be obtained.

\subsection{Analysis of the $\Phi^{4}$ model}

Let us focus now on (2.9) with $c_{2}=1$ since the KPZ equation has non-generic additional structure which makes it amenable to alternative techniques [GJ14, GP18]. We are therefore interested in the study of the family of solutions to

$$
\partial_{t} \Phi=\Delta \Phi+c \Phi-\Phi^{3}+\xi
$$

with $c \in \mathbf{R}$. The problem is that solutions to the stochastic heat equation

$$
\partial_{t} X=\Delta X+\xi
$$


in three dimensions (and therefore presumably also solutions to (3.1), whatever meaning we choose to give to the equation) are random distributions of order $-1 / 2$. In other words, the operation $\Phi \mapsto \Phi^{3}$ is not closable on any classical space of functions / distributions containing the solutions to (3.1). The idea then is to enrich this space in a way that incorporates the minimal amount of information required in order to be able to realise $\Phi \mapsto \Phi^{3}$ as a continuous map, but in a way that remains consistent with the classical interpretation of (2.10), so that one has hope to be able to obtain the type of universality results mentioned above.

A simple, but surprisingly effective, idea introduced in this context in [DPD02, DPDo3] (but see also [Bou96] for the same idea in a slightly different context) is to try to solve (3.1) by setting $\Phi=\Psi+X$, where $X$ is given by (3.2), so that it remains to solve

$$
\partial_{t} \Psi=\Delta \Psi+c(\Psi+X)-\left(\Psi^{3}+3 X \Psi^{2}+3 X^{2} \Psi+X^{3}\right) .
$$

The point here is that $\xi$ no longer appears explicitly in the right hand side, so we can expect $\Psi$ to be more regular than $\Phi$, hopefully function-valued so that the terms $\Psi^{2}$ and $\Psi^{3}$ are well-defined.

Assume now that $X$ is scaling invariant with some negative scaling exponent $-\alpha$ (in dimension $d$, this is the case for the solution to (3.2) with $\alpha=\frac{d}{2}-1$ ). Then, even though we don't know a priori how to define its powers, one would expect any reasonable definition of $X^{p}$ to be a stationary process which is scaling invariant with exponent $-\alpha p$. Under relatively weak moment assumptions, such processes belong to the negative Hölder spaces $\mathscr{C}^{\beta}$ for all $\beta<-\alpha p$, which suggests that the least regular term on the right hand side of (3.3) is the term $X^{3}$, with regularity just below $-3 \alpha$, so one would expect by Schauder's estimate $\Psi$ to have regularity just below $2-3 \alpha$, which is positive as soon as $\alpha<2 / 3$. Since the product of a function in $\mathscr{C}^{\gamma}$ with a distribution in $\mathscr{C}^{\beta}$ is well-defined (in the sense that there is a unique continuous bilinear extension of the usual product) if and only if $\beta+\gamma>0$, this shows that, assuming that $X^{2}$ and $X^{3}$ are known, (3.3) is well-posed as soon as $2-5 \alpha>0$, i.e. $\alpha<2 / 5$.

In dimension $d=2$, the case treated in [DPDo3], this is indeed the case. However, it is still not clear how the processes $X^{2}$ and $X^{3}$ should be defined in that case. A "naive" construction of these processes would be to try to consider a regularisation $X_{\varepsilon}$ of $X$ and to check whether $X_{\varepsilon}^{2}$ and $X_{\varepsilon}^{3}$ have a limit. Write for example $\xi_{\varepsilon}$ for a centred Gaussian random field with covariance given by $\varrho_{\varepsilon}(t, x)=\varepsilon^{-3} \varrho\left(t / \varepsilon^{2}, x / \varepsilon\right)$ for some compactly supported function $\varrho$ integrating to 1 and define $X_{\varepsilon}$ by $(3.2)$ with $\xi$ replaced by $\xi_{\varepsilon}$. It can then be verified that

$$
\mathbf{E} X_{\varepsilon}^{2}=\int P(t, x) P(s, y) \varrho_{\varepsilon}(t-s, y-x) d t d s d x d y,
$$


where $P$ is the standard heat kernel. A simple calculation shows that, in dimension $d=2$, one has $\mathbf{E} X_{\varepsilon}^{2} \sim \log \varepsilon$, while in dimension $d=3$ one has $\mathbf{E} X_{\varepsilon}^{2} \sim \varepsilon^{-1}$, so that there is no hope that $X_{\varepsilon}^{2}$ converges to a limiting proces as $\varepsilon \rightarrow 0$.

However, setting $C_{\varepsilon}=\mathbf{E} X_{\varepsilon}^{2}$, it turns out that the Wick powers $X_{\varepsilon}^{\diamond p} \stackrel{\text { def }}{=}$ $H_{p}\left(X_{\varepsilon}, C_{\varepsilon}\right)$, where $H_{p}$ denotes the $p$ th Hermite polynomial do converge to a limit for $p$ small enough. Indeed, writing $G_{\varepsilon}$ for the covariance of $X_{\varepsilon}$, namely

$$
G_{\varepsilon}(t, x)=\mathbf{E} X_{\varepsilon}(0,0) X_{\varepsilon}(t, x),
$$

one can verify that the covariance of $X_{\varepsilon}^{\diamond p}$ is simply given by $G_{\varepsilon}^{p}$. If $X$ is self-similar with exponent $-\alpha$, then its covariance is homogeneous of order $-2 \alpha$, so that $G_{\varepsilon}^{p}$ does indeed converge to $G^{p}$ in distribution, provided that $2 p \alpha<d+2$. (The reason why we have $d+2$ appearing here is that this is precisely the threshold at which a homogeneous function with parabolic scaling loses integrability at the origin.) Recalling that $\alpha=\frac{d}{2}-1$, we conclude that $X_{\varepsilon}^{\diamond 2}$ and $X_{\varepsilon}^{\diamond 3}$ do indeed converge to non-trivial limits $X^{\diamond 2}$ and $X^{\diamond 3}$ in dimension $d<4$.

In dimension $d=2$, it is therefore natural to define $\Phi_{\varepsilon}=\Psi_{\varepsilon}+X_{\varepsilon}$ with $\Psi_{\varepsilon}$ defined as in (3.3), but with $X^{2}$ and $X^{3}$ replaced by $X_{\varepsilon}^{\diamond 2}$ and $X_{\varepsilon}^{\diamond 3}$. Standard continuity estimates then show that $\Phi_{\varepsilon}$ converges to some limit $\Phi$, which we would like to interpret as a "solution" to (3.1). This raises the question of the interpretation of the resulting process, in particular whether it is possible to obtain it as a limit of solutions to stochastic PDEs of the type (3.1) with the noise replaced by a smoothened version. For this, note that $\Psi_{\varepsilon}$ solves

$$
\begin{aligned}
\partial_{t} \Psi_{\varepsilon} & =\Delta \Psi_{\varepsilon}+c\left(\Psi_{\varepsilon}+X_{\varepsilon}\right)-\left(\Psi_{\varepsilon}^{3}+3 X_{\varepsilon} \Psi_{\varepsilon}^{2}+3 X_{\varepsilon}^{\diamond 2} \Psi_{\varepsilon}+X_{\varepsilon}^{\diamond 3}\right) \\
& =\Delta \Psi_{\varepsilon}+c\left(\Psi_{\varepsilon}+X_{\varepsilon}\right)-\left(\Psi_{\varepsilon}^{3}+3 X_{\varepsilon} \Psi_{\varepsilon}^{2}+3\left(X_{\varepsilon}^{2}-C_{\varepsilon}\right) \Psi_{\varepsilon}+\left(X_{\varepsilon}^{3}-3 C_{\varepsilon} X_{\varepsilon}\right)\right) \\
& =\Delta \Psi_{\varepsilon}+\left(c+3 C_{\varepsilon}\right)\left(\Psi_{\varepsilon}+X_{\varepsilon}\right)-\left(\Psi_{\varepsilon}^{3}+3 X_{\varepsilon} \Psi_{\varepsilon}^{2}+3 X_{\varepsilon}^{2} \Psi_{\varepsilon}+X_{\varepsilon}^{3}\right)
\end{aligned}
$$

This shows that, for any fixed $\varepsilon>0, \Phi_{\varepsilon}$ is indeed nothing but the solution to (3.1) with $\xi$ replaced by $\xi_{\varepsilon}$ and $c$ replaced by $c+3 C_{\varepsilon}$.

\subsection{Renormalisation}

Let us step back for a moment and try to give a high-level overview of what's happening here. We started from a formal expression $(3.1)$ which hints at the existence of a family of processes $\Phi^{c}$ indexed by a parameter $c$. We then consider some natural approximation $\Phi_{\varepsilon}^{c}$ of these processes and study it for small $\varepsilon$. Unfortunately, for every fixed $c$, the sequence $\Phi_{\varepsilon}^{c}$ diverges (or rather converges to something trivial in this particular case [HRW 12] ) as $\varepsilon \rightarrow 0$. However, the family of processes $\left\{\Phi_{\varepsilon}^{c}\right\}_{c \in \mathbf{R}}$ does converge to a limiting family $\left\{\Phi^{c}\right\}_{c \in \mathbf{R}}$ as $\varepsilon \rightarrow 0$. This is so because, when considering the whole family of processes as our object of interest, we do not care about the way in which it is parametrised. We can therefore 
just as well reparametrise it by viewing it as the family $\left\{\Phi_{\varepsilon}^{c+C_{\varepsilon}}\right\}_{c \in \mathbf{R}}$ with $C_{\varepsilon}$ as in the previous section.

This idea first arose in theoretical physics in the earlier part of the 2oth century during the development of quantum field theory. The situation there is similar: one starts from a family of formal expressions for the Lagrangian of a system and then tries to use this in order to compute scattering amplitudes. These amplitudes are expressed as an infinite series of terms, each of which is described by a Feynman diagram. Some of these diagrams happen to encode diverging integrals and are therefore not well-defined. The procedure that was eventually devised in order to "cure" these divergencies can be summarised as follows:

- Construct a family $M_{\varepsilon}^{c}$ of theories indexed by the same constants $c$ appearing in the original formal Lagrangian, as well as on some cut-off parameter $\varepsilon$. These theories are such that they give rise to well-defined Feynman diagrams for any fixed $\varepsilon>0$ and they formally appear to approximate to the desired theory as $\varepsilon \rightarrow 0$.

- Find an $\varepsilon$-dependent reparametrisation $g^{\varepsilon}$ of the parameter space in such a way that, for every $c, M_{\varepsilon}^{g^{\varepsilon} c}$ converges to a non-trivial limiting theory as $\varepsilon \rightarrow 0$.

- Show that, modulo reparametrisation of its parameter space, the family of limits obtained in this way does not depend on the regularisation procedure used in the first step.

Whenever we are in such a situation, we call the theories $\hat{M}^{c}$ and $\hat{M}_{\varepsilon}^{c}$ given by

$$
\hat{M}^{c}=\lim _{\varepsilon \rightarrow 0} \hat{M}_{\varepsilon}^{c}, \quad \hat{M}_{\varepsilon}^{c}=M_{\varepsilon}^{g^{\varepsilon} c},
$$

the "renormalised theories". In the context of QFT, the arguments $c$ parametrising the renormalised theories are called the "effective coupling constants", while the corresponding arguments $g^{\varepsilon} c$ for the regularised theory are called the "bare coupling constants".

This turns out to be a rather generic state of affairs which also applies mutatis mutandis in the context of semilinear stochastic PDEs, where the $M^{c}$ 's are solution theories to families of such equations.

\subsection{A simple example}

Before we proceed, let us now give a very simple and self-contained example of renormalisation. In this example, which does not at all have the pretense to be connected to physical reality in any sense, a "theory" $M$ is given by a Schwartz distribution, i.e. a continuous linear map $M: \mathcal{S} \rightarrow \mathbf{R}$, where $\mathcal{S}$ denotes the space 
of Schwartz functions. Assume now that we would like to describe the family of Schwartz distributions formally given by $M^{c}(x)=\frac{c_{1}}{|x|}-c_{2} \delta_{0}(x)$. This is of course nonsensical since, for every test function $\varphi$ with $\varphi(0) \neq 0$, one would then have

$$
M^{c}(\varphi)=\int_{\mathbf{R}} c_{1} \frac{\varphi(x)}{|x|} d x-c_{2} \varphi(0)=\infty,
$$

so that $M^{c}$ really isn't a Schwartz distribution at all!

Given a continuous function $\eta:[-1,1] \rightarrow \mathbf{R}$ with $\eta(-1)=\eta(1)=1$, we have a natural way of regularising this theory by replacing the singular function $1 /|x|$ by the continuous function given by

$$
\frac{1}{|x|_{\varepsilon}}:=\left\{\begin{array}{cc}
1 /|x| & \text { if }|x| \geq \varepsilon \\
\varepsilon^{-1} \eta(x / \varepsilon) & \text { otherwise }
\end{array}\right.
$$

This regularisation procedure then yields a family $M_{\varepsilon}^{c, \eta}$ of well-defined Schwartz distributions by simply replacing $|x|$ by $|x|_{\varepsilon}$ in (3.5). In order to obtain finite quantities in the limit $\varepsilon \rightarrow 0$, we then set

$$
c_{1}=\hat{c}_{1}, \quad c_{2}=\hat{c}_{1} \int_{-1}^{1} \frac{d x}{|x|_{\varepsilon}}+\hat{c}_{2}=\hat{c}_{1}\left(\int \eta(x) d x-2 \log \varepsilon\right)+\hat{c}_{2} .
$$

In this way, we have

$$
M_{\varepsilon}^{c(\varepsilon, \eta, \hat{c}), \eta}(\varphi)=\hat{c}_{1} \int_{|x| \geq 1} \frac{\varphi(x)}{|x|} d x+\hat{c}_{1} \int_{|x|<1} \frac{\varphi(x)-\varphi(0)}{|x|_{\varepsilon}} d x-\hat{c}_{2} \varphi(0) .
$$

It is now immediate that this expression does indeed have a limit as $\varepsilon \rightarrow 0$ and that this limit is indeed independent of the choice of regularisation function $\eta$ :

$$
\hat{M}^{\hat{c}}(\varphi)=\hat{c}_{1} \int_{\mathbf{R}} \frac{\varphi(x)-\mathbf{1}_{|x|<1} \varphi(0)}{|x|} d x-\hat{c}_{2} \varphi(0) .
$$

(But for this we had to adjust the "bare" parameter $c_{2}$ in an $\eta$-dependent way!)

\subsection{Renormalisation of SPDEs}

Let us now give an overview of how the procedure explained in Section 3.2 below is implemented in the context of stochastic PDEs. Consider a family of equations of the type

$$
\partial_{t} u_{i}=L u_{i}+F_{i}^{(0)}(u)+\sum_{j=1}^{m} F_{i}^{(j)}(u) \xi_{j}
$$

where $L$ is a strictly elliptic differential operator with constant coefficients (for example $L=\Delta$ or $L=-\Delta^{2}$ ), the $\xi_{j}$ are finitely many stationary random distributions which, at small scales, are close to scale invariant with exponents $\alpha_{j}$, and 
the $F_{i}^{(j)}$ are local functions of $u$, in the sense that $F_{i}^{(j)}(u)(t, x)$ only depends on $u$ and its derivatives evaluated at $(t, x)$. We furthermore assume that this system of equations is locally subcritical. We do not give a precise definition of what this means, loosely speaking it means that the scaling behaviour of the non-linear terms appearing on the right hand side of $(3.6)$ is dominated at small scales by that of the noise terms, in the sense of formal powercounting. It is a purely combinatorial condition, which only depends on the order of $L$, the exponents $\alpha_{j}$, and the degrees of the nonlinearities $F_{i}^{(j)}$ (with the convention that arbitrary smooth functions count as polynomials of infinite degree).

For example, in the case of the (2.9), the noise term in the right hand side is $\xi$, which is scale invariant (under parabolic scaling) with exponent $-\frac{d+2}{2}$ in the sense that

$$
\varepsilon^{\alpha} \xi\left(t / \varepsilon^{2}, x / \varepsilon\right) \stackrel{\text { law }}{=} \xi(t, x), \quad \alpha=-\frac{d+2}{2} .
$$

On the other hand, the "worst" nonlinear term is $\Phi^{3}$ which is expected to be dominated at small scales by a scale-invariant behaviour with exponent $-3 \beta$, where $\beta=\frac{d-2}{2}$. This is because the solution to $\partial_{t} \Phi=\Delta \Phi+\xi$ displays is selfsimilar of order $\beta$ as a consequence of (3.7) and self-similarity exponents do add up when multiplying terms. (Or at least they would if these were functions that we are allowed to just multiply together!)

We see that $-3 \alpha>-\frac{d+2}{2}$ if and only if $d<4$, so that this equation is locally subcritical in dimensions below 4 , and we say that its "critical dimension" is $d=4$. Note that this terminology is to some extent compatible with the one found in the physics literature, according to which the critical dimension of the Ising model is also 4. Performing the analogous powercounting for the KPZ equation shows that it is locally subcritical for $d<2$. The following is a synthesis of the main results of [Hai14, BHZ16, $\left.\mathrm{CH}_{16}, \mathrm{BCCH}_{17}\right]$ :

Theorem 3.1 Consider (3.6) on a bounded d-dimensional torus and, for any smooth noise $\xi^{(\varepsilon)}$, write $\left.\overline{M(F,} \xi^{(\varepsilon)}\right)$ for the classical solution to 3.6 with locally subcritical nonlinearity $F$ and noise $\xi$.

Then, there exists a finite-dimensional Lie group $\mathfrak{R}$ acting on the space of nonlinearities as well as a map $\xi \mapsto g^{\xi} \in \mathfrak{R}$ defined on centred smooth stationary noises satisfying suitable moment conditions such that the map

$$
(F, \xi) \mapsto \mathcal{M}\left(g^{\xi} F, \xi\right),
$$

extends continuously to all noises compatible with the scaling exponents $\alpha_{j}$.

The formulation of this result is somewhat imprecise on purpose. A precise formulation requires giving a formal definition of local subcriticality, a precise definition of a class of admissible noises, as well as a topology in which they can 
be approximated. This is surprisingly subtle and technical, so we avoid giving these details here. For the same reason, we also ignored the question of a suitable class of initial conditions. In the cases discussed in these notes, one can find $\alpha \in \mathbf{R}$ such that solutions exists for all $u_{0} \in \mathscr{C}^{\alpha}$, and such that these solutions then also take values in $\mathscr{C}^{\alpha}$ for positive times. This is however not the case in general: there are situations in which solutions can only be defined for initial conditions that are "sufficiently close to equilibrium" in a sense that can be made precise. This is the case for example when considering a model analogous to (2.9), but in "effective dimension" $d=4-\delta$ for small enough. One way to set up such a model is to consider (2.9) in dimension 4, but to replace $\xi$ by space-time white noise convolved with a kernel of the type $(t, x) \mapsto\left(t^{2}+|x|^{4}\right)^{\frac{\delta-6}{4}}$, where the number 6 refers to the scaling dimension of $4+1$-dimensional parabolic space-time. Finally, this general result is local in time, i.e. we work in a topology that allows solutions to blow up in finite time and all approximation results are only relevant up to this blow-up time.

\subsection{Structure of proof}

Let us now give a short overview of how a result like Theorem 3.1 can be proven. The first step, which is based on an idea first introduced by Lyons in the nineties in the context of controlled ODEs (and in particular stochastic differential equations, see [Ly098, LCL07, LQ02, FV10, FH14]]) is to factorise the classical solution map $\mathcal{U}$ into a map that acts only on the noise and "enhances" it with additional information and a map that makes uses of this additional information to build the solution. In the context of ODEs, this enhancement of the noise is called a "rough path", while in the present context it is called a "model" for reasons that will become clearer later on.

For definiteness, let us write $\mathcal{N}$ for a suitable space of admissible nonlinearities leading to locally subcritical problems and $\mathcal{Y}$ for a suitable space of distributions including possible blow-up times arising in Theorem 3.1. so that the classical solution map $\boldsymbol{M}$ can be viewed as a map $\boldsymbol{M}: \mathcal{N} \times \mathscr{B}_{\infty} \rightarrow \mathcal{Y}$, where the second factor $\mathscr{B}_{\infty}$ is a space of smooth noises. We also write $\mathscr{B}$ for the space of all distributions $\left(\xi_{i}\right)_{i}$ such that $\xi_{i} \in \mathscr{C}^{\alpha_{i}-\kappa}$ for some (small) $\kappa>0$ and every $i \in\{1, \ldots, m\}$, with $\alpha_{i}$ as in $\left(3.6\right.$. We also view $\mathscr{B}_{\infty}$ as a subspace of $\mathscr{B}$. From now on we completely ignore the additional dependence of the solution on the initial condition since this does not introduce any additional conceptual difficulty.

The first step mentioned above is thus to find a topological space $X$ and maps $\mathscr{L}: \mathscr{B}_{\infty} \rightarrow \mathscr{X}, \pi: X \rightarrow \mathscr{B}$ and $\mathscr{M}_{A}: \mathcal{N} \times \mathcal{X} \rightarrow \mathcal{Y}$ such that one has the identities

$$
\mathcal{M}(F, \xi)=\mathscr{M}_{A}(F, \mathscr{L} \xi), \quad \pi \circ \mathscr{L}=\mathrm{id} .
$$

Without additional constraints, it is of course trivial to do so: just take $\mathcal{X}=\mathscr{B}_{\infty}$ and $\mathscr{L}$ and $\pi$ to be the identity. It is however highly non-trivial to do this if we impose the following additional constraints: 
1. The map $M_{A}$ is continuous.

2. The map $\pi$ is continuous and surjective.

Indeed, these conditions are competing: the smaller the space $\mathcal{X}$, the easier it is for $M_{A}$ to be continuous, but it needs to be large enough for $\pi$ to be surjective. As a matter of fact, it was shown in [Lyo91] that already in very simple cases it is impossible for any Banach space $X$ to simultaneously satisfy all these constraints!

It was shown in [Hai14] (see also [BHZ16] for a cleaner formulation that allows for systems of equations and collections of noises with different scaling exponents) that it is indeed possible to find such a space $\mathscr{X}$ in all locally subcritical situations. The problem is that the map $\mathscr{L}$, although continuous on the space of smooth functions, does of course not extend in a continuous way to all of $\mathscr{B}$, so that this is not yet sufficient to give a canonical interpretation to solutions to 3.6 : we would like to find a "canonical" random variable $Z$ with values in $X$ such that the law of $\pi Z$ is equal to that of the noise $\xi$, so that we could then define solutions to (3.6) to be given by $\mu_{A}(F, Z)$. Ideally, one would even want these random variables to be defined on the same probability space, so that $Z=\hat{\mathscr{L}}(\xi)$ for some measurable map $\hat{\mathscr{L}}$ satisfying $\pi \circ \hat{\mathscr{L}}=$ id, which would then yield a corresponding notion of "strong solution".

It was shown in [Hai14] that there exists a finite-dimensional nilpotent Lie group $\Re$ acting on $\mathscr{X}$ in a way that leaves $\pi$ invariant in the sense that $\pi(g Z)=\pi(Z)$ for every $Z \in X$ and every $g \in \mathfrak{R}$. It is therefore natural to try to build the random variable $Z$ by considering a regularisation $\xi^{(\varepsilon)}$ of the noise $\xi$ and setting $Z^{(\varepsilon)}=g^{\varepsilon} \xi^{(\varepsilon)}$. The question is then whether there exist elements $g^{\varepsilon}$ such that the $Z^{(\varepsilon)}$ converge. In [Hai14], this was shown for two examples on a case-by-case basis, but a general theory was lacking due to a lack of understanding of the group $\mathfrak{R}$ and its action on $\mathcal{X}$.

This was partly remedied in [BHZ16], where an explicit description of $\mathfrak{R}$ (or rather a "sufficiently large" subgroup thereof) is given. This allows [CH16] to show the following:

Proposition 3.2 In the context of Theorem 3.1 there exists a map $\xi \mapsto g^{\xi} \in \Re$ defined on centred smooth stationary noises satisfying suitable moment conditions such that the map

$$
\xi \mapsto g^{\xi} \mathscr{L}(\xi),
$$

extends continuously to all noises compatible with the scaling exponents $\alpha_{j}$.

Remark 3.3 Note that this is a probabilistic statement: it is important to consider random noises $\xi$ that are stationary in space-time. The continuity mentioned in the statement is not a continuity at the level of samples of this process, but at the 
level of its law. When considering convergences of the type $\varrho_{\varepsilon} \star \xi \rightarrow \xi$ for some mollifier $\varrho_{\varepsilon}$, the whole sequence naturally lives on the same probability space and the result of [ $\mathrm{CH} 16]$ actually yields convergence in probability.

Remark 3.4 The map $\xi \mapsto g^{\xi}$ is constructed as follows. Given any class of locally subcritical stochastic PDEs as described above, one first builds a linear space $\hat{X}$ as well as a polynomial (in the sense of "finite sum of multilinear") map $\hat{\mathscr{L}}: \mathscr{B}_{\infty} \rightarrow \hat{\mathscr{X}}$ which is such that it maps stationary processes to stationary processes under a suitable action of the group of translations on $\hat{x}$. The actual (nonlinear) space $\mathcal{X}$ can then be viewed as a subset of $\hat{X}$ which turns into a Polish space when endowed with a suitable metric. This metric is not derived from any norm on $\hat{x}$ but instead encodes the nonlinear features of $\mathscr{X}$. The map $\mathscr{L}$ mentioned in the proposition then coincides with $\hat{\mathscr{L}}$ and the action of $\mathfrak{R}$ on $X$ is actually derived from an action on $\hat{X}$. The element $g^{\xi}$ is then the unique element of $\mathfrak{R}$ with the property that

$$
\mathbf{E}\left(g^{\xi \hat{\mathscr{L}}}(\xi)\right)(0)=0
$$

The fact that such an element exists, is unique, that the action of $\mathfrak{R}$ on $\hat{X}$ leaves $\mathscr{X}$ invariant and that it is continuous in the topology of $X$ are all non-trivial statements that are contained in [Hai14] for special cases and [BHZ16] in full generality.

When combining this with the continuity of the map $\Omega_{A}$, this shows that the map $\hat{M}$ defined by

$$
\hat{M}(F, \xi)=M_{A}\left(F, g^{\xi} \mathscr{L}(\xi)\right)
$$

does indeed have the type of continuity properties with respect to the noise that are announced in Theorem 3.1 . The last missing ingredient in the proof is the fact that $\hat{M}(\cdot, \xi)$ is really nothing but a reparametrisation of $M(\cdot, \xi)$. This is shown in full generality in [BCCH17]:

Proposition 3.5 In the context of Theorem 3.1 there exists an action of $\Re$ onto $X$ on the right such that, for every $Z \in X$ and every $F \in \mathcal{N}$, the identity

$$
\boldsymbol{M}_{A}(F, g Z)=M_{A}(F g, Z) .
$$

holds for every $g \in \mathfrak{R}$. In particular, it follows that the renormalised solution map $\hat{M}$ is related to the classical solution map by $\hat{M}(F, \xi)=M\left(F g^{\xi}, \xi\right)$.

\section{Regularity structures}

In this final section, we give a description of the main ingredients appearing in the sketch of proof given in the previous section, namely the space $X$ and the group $\mathfrak{R}$. 


\subsection{Revisiting the $\Phi^{4}$ equation}

Let us first note that the construction given in Section 3.1 is precisely of the type described in the previous section with the important feature that in this case the space $X$ itself is linear. Indeed, we can set

$$
\mathfrak{X}=\mathscr{C}^{-\alpha} \oplus \mathscr{C}^{-2 \alpha} \oplus \mathscr{C}^{-3 \alpha},
$$

as well as

$$
\mathscr{L}(\xi)=\left(X, X^{2}, X^{3}\right),
$$

where $X$ is given as the solution to $\partial_{t} X=\Delta X+\xi$. (We ignore the minor subtleties arising from the fact that such a solution may not necessarily exist. One way of circumventing this problem is to actually set $\partial_{t} X=(\Delta-1) X+\xi$ which leads to minor modifications in the formulas given below.) Conversely, we set

$$
\pi\left(X_{1}, X_{2}, X_{3}\right)=\partial_{t} X_{1}-\Delta X_{1} .
$$

The "solution map" $\varkappa_{A}$ is then given by postulating that, for $Z=\left(X_{1}, X_{2}, X_{3}\right)$, $\Phi=\mathcal{M}_{A}(c, Z)$ is given by $\Phi=\Psi+X_{1}$, with $\Psi$ solving

$$
\partial_{t} \Psi=\Delta \Psi+c\left(\Psi+X_{1}\right)-\left(\Psi^{3}+3 X_{1} \Psi^{2}+3 X_{2} \Psi+X_{3}\right) .
$$

Comparing this to $(\overline{3 \cdot 3})$, we immediately conclude that $(3.8)$ does indeed hold.

Let now $\mathfrak{R}=\left(\mathbf{R}^{2},+\right)$ and, for $g \in \mathbf{R}^{2}$, define its action on $\mathcal{X}$ by

$$
g Z=\left(X_{1}, X_{2}-g, X_{3}-3 g X_{1}\right) .
$$

The calculation in (3.4) then shows that the identity $(\sqrt{3.10})$ does hold, provided that the action of $\mathfrak{R}$ onto the space of right hand sides (which in this case is parametrised by the constant $c$ ) is given by $g: c \mapsto c+3 g$. The map $\xi \mapsto g^{\xi}$ is then given by

$$
g^{\xi}=\mathbf{E} X^{2}(0), \quad \partial_{t} X=\Delta X+\xi .
$$

If we restrict ourselves to symmetric noises, or even just to noises with vanishing first and third moments, it immediately follows that this choice of $g^{\xi}$ does indeed satisfy (3.9). The fact that it also makes $\xi \mapsto g^{\xi} \mathscr{L}(\xi)$ continuous is less obvious and relies on the particular form of the action (4.2): the constant 3 appearing in the last component seems somewhat arbitrary, but it serves to seemingly unrelated purposes. First, if we restrict ourselves to actions of the form (4.2), the value 3 for this constant is the only possible choice which guarantees that there exists a dual action of $\mathfrak{R}$ on the space of right hand sides for our equation such that $(3.10)$ holds. The second consequence of this particular choice is that $\xi \mapsto g^{\xi} \mathscr{L}(\xi)$ is continuous provided that we restrict ourselves to stationary processes satisfying a natural cumulant bound. 
Although we will not give a proof of this latter fact here, let's perform a calculation that makes it plausible, and that simultaneously shows what kind of probabilistic assumptions one should impose on the noise $\xi$. Fix a test function $\varphi$ and consider the random variables

$$
\hat{Y}_{\varphi}=\int \varphi(z) X(z) d z, \quad Y_{\varphi}=\int \varphi(z)\left(X^{2}(z)-\mathbf{E} X^{2}(z)\right) d z
$$

with $X$ given by (3.2). Since $X$ is stationary, $\mathbf{E} X^{2}(z)=\mathbf{E} X^{2}(0)$ and these are precisely the random variables obtained by testing the first two components of $g^{\xi} \mathscr{L}(\xi)$ with $\varphi$.

We now give an example in dimension 3 of a sequence of non-Gaussian processes $\xi_{\varepsilon}$ so that the corresponding random variables $Y_{\varphi}^{(\varepsilon)}$ and $\hat{Y}_{\varphi}^{(\varepsilon)}$ converge to a limit, but so that $\mathbf{E} X_{\varepsilon}^{2}(z)$ diverges. For this, let $\eta$ be a stationary generalised Gaussian random field with covariance given by $\mathbf{E} \eta(z) \eta\left(z^{\prime}\right)=K\left(z-z^{\prime}\right) \sim$ $\left|z-z^{\prime}\right|^{-\frac{9}{4}}$, with $|z|$ denoting the parabolic norm of $z=(t, x)$. We then set $\eta_{\varepsilon}=\varrho_{\varepsilon} \star \eta$, write $K_{\varepsilon}$ for the covariance function of $\eta_{\varepsilon}$, and set $\xi_{\varepsilon}(z)=\eta_{\varepsilon}^{2}(z)-K_{\varepsilon}(0)$.

We now introduce a graphical notation for integrals involving $K_{\varepsilon}$, the heat kernel $P$, and the test function $\varphi$. We denote integration variables by nodes of our graphs, with the origin drawn as a special node $\bullet$. Each line then represents a kernel, with $\longrightarrow$ representing the heat kernel $P, \ldots \ldots \ldots$ representing the covariance $K_{\varepsilon}$, and $\longrightarrow$ representing the test function $\varphi$. The argument of a kernel is the difference between the values of the variables represented by the corresponding target and base nodes. The line representing $K_{\varepsilon}$ are not oriented because this kernel is symmetric, being the covariance of a stochastic process. With this notation, setting for example

$$
\hat{Y}_{\varphi}=\int \varphi(z) X(z) d z
$$

it follows from the definition of $X$ and Wick's formula that

$$
\mathbf{E} \hat{Y}_{\varphi}^{2}=2 \int \varphi\left(z_{1}\right) \varphi\left(z_{2}\right) P\left(z_{1}-z_{3}\right) P\left(z_{2}-z_{4}\right) K_{\varepsilon}\left(z_{3}-z_{4}\right)^{2} d z=2\lfloor\cdots \cdots \cdot .
$$

Note now that in dimension $3+1$ with parabolic scaling, if $K_{i}$ are kernels behaving like $K_{i}(z) \sim|z|^{-\alpha_{i}}$ (we say that they are self-similar of order $\alpha_{i}$ ) and one has $\alpha_{i}<5$ while $\alpha_{1}+\alpha_{2}>5$, then $K_{1} \star K_{2}$ is a kernel which is again self-similar of order $\alpha_{1}+\alpha_{2}-5$. In this particular example, $K_{\varepsilon}^{2}$ is self-similar of order $\frac{9}{2}$, while the heat kernel is self-similar of order 3 , so that the kernel

$$
\bar{P} \star K_{\varepsilon}^{2} \star P=\longleftarrow \cdot \cdots \cdot
$$


where $\bar{P}(z)=P(-z)$, is self-similar of order $\frac{1}{2}$ and therefore locally integrable. A similar calculation allows to conclude that $\lim _{\varepsilon \rightarrow 0} \mathbf{E}\left(\hat{Y}_{\varphi}^{(\varepsilon)}-\hat{Y}_{\varphi}\right)^{2}=0$ as claimed.

For this, no renormalisation was necessary. The situation gets a bit more interesting regarding $Y_{\varphi}$. It follows from Wick's formula combined with the definition of $\xi_{\varepsilon}$ that one has

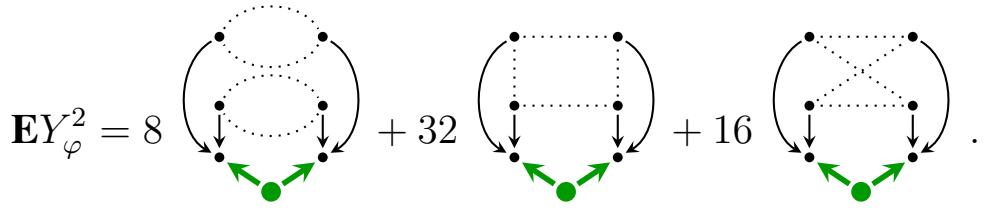

The main point here is that thanks to the fact that we subtracted $\mathbf{E} X^{2}(0)$ in the definition of the second component, there is no term of the form

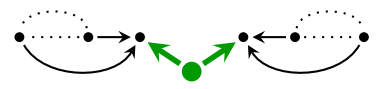

appearing in this expression. Instead, all the terms appearing in (4.3) are 2connected: one needs to cut at least two edges in order to disconnect them. The study of the convergence of multiple integrals of this type is a standard ingredient of perturbative quantum field theory. In this case, one can apply Weinstein's theorem [Wei60] (see also [Hai17, Prop. 2.3]) which can be formulated as follows. Assign to each edge $e$ an exponent $a_{e}$ by setting $a_{e}=0$ for edges representing $\varphi, a_{e}=3$ for edges representing $P$, and $a_{e}=\frac{9}{4}$ for edges representing $K_{\varepsilon}$. Then, integrals described by a graph $\Gamma$ as above converge if, for every subgraph $(\bar{V}, \bar{E}) \subset \Gamma$ (with $\bar{V}$ a subset of the vertices of $\Gamma$ and $\bar{E}$ a subset of its edges such that the endpoints of every edge in $\bar{E}$ belongs to $\bar{V}$ ), one has the bound

$$
\sum_{e \in \bar{E}} a_{e}<5(|\bar{V}|-1)
$$

One can verify that all the graphs appearing in (4.3) do indeed have this property while the graph (4.4) does not. (Just take as a subgraph the graph $\bullet$ which one has $\sum_{e \in \bar{E}} a_{e}=10 \frac{1}{2}>10$.)

What we've learned from this calculation is that the key property of the renormalisation procedure (3.9) is that it creates cancellations for terms of lower connectivity in the graphical representation for the variances of the random variables $Y_{\varphi}$ and $\hat{Y}_{\varphi}$. In general, one has a decomposition of the type (4.3), but with instances of $2 \cdot \cdots \cdot$ replaced by the covariance of $\xi_{\varepsilon}$ and instances of $32^{\bullet} \cdot \ldots+16^{\bullet}$ replaced by its four-point cumulant. It is then natural to impose conditions on these cumulants that are akin to what we observed above. The key condition one imposes is that for $k=\ell+m$ and writing $\kappa_{k}$ for the $k$-point cumulant, the behaviour of $\kappa_{\ell+m}\left(z_{1}, \ldots, z_{\ell}, z_{1}^{\prime}, \ldots, z_{m}^{\prime}\right)$ is less singular than that of 
$\kappa_{\ell}\left(z_{1}, \ldots, z_{\ell}\right) \kappa_{m}\left(z_{1}^{\prime}, \ldots, z_{m}^{\prime}\right)$ as some of the variables $z_{i}$ get close to each other. In other words, one assumes that cumulants behave "better" than what one can deduce from their expressions in terms of moments. This is a very natural condition which, as we have just seen in an example, is verified for very large classes of random fields.

Remark 4.1 One can also consider noises with non-vanishing third moments, but this then forces us to consider a slightly larger class of right hand sides, for example we can consider all equations of the type

$$
\partial_{t} \Phi=\Delta \Phi+c_{0}+c_{1} \Phi+c_{2} \Phi^{2}-\Phi^{3}+\xi
$$

Furthermore, one should then take $\mathfrak{R}=\left(\mathbf{R}^{2},+\right)$ acting on the space $\mathscr{X}$ by

$$
g Z=\left(X_{1}, X_{2}-g_{1}, X_{3}-3 g_{1} X_{1}-g_{2}\right) .
$$

In this case, it is a good exercise to show that the dual action on 4.6 for which (3.10) holds is given by

$$
\left(c_{0}, c_{1}, c_{2}\right) \mapsto\left(c_{0}-c_{2} g_{1}+g_{2}, c_{1}+3 g_{1}, c_{2}\right) .
$$

\subsection{Basic definitions}

In the above example, the space $\mathcal{X}$ is a Banach space and the group $\mathfrak{R}$ is abelian. At the level of generality considered in Theorem 3.1 , it is typically not possible to enforce this and a more sophisticated approach is required.

The problem is that in general, the trick of subtracting some fixed process from the solution in order to improve its regularity properties does not work. The idea then is to perform a similar procedure, but to proceed locally rather than globally and to determine these local terms in a self-consistent way. More precisely, the aim is to provide a local description of the solution by a kind of Taylor expansion and to then find a fixed point problem for the coefficients of this expansion. The usual Taylor polynomials won't do of course since, as we have already seen above, solutions may not even be function-valued.

Instead, as in the previous subsection, the idea is to build a collection of noisedependent objects (in this case $X, X^{2}$ and $X^{3}$ ) which are useful in order to describe both the solution to 3.6 and its right hand side. In general, these objects can be arranged into a structure that closely mimics that of the usual Taylor polynomials. Before we proceed, we describe this structure in a very general context that does not refer to stochastic PDEs at all.

The starting point for our construction is a vector space $T$ that should be though of as containing the coefficients of our "Taylor-like" expansion at any point. It is natural to postulate that $T$ is a graded vector space $T=\bigoplus_{\alpha \in A} T_{\alpha}$, for some 
discrete set $A$ of possible "homogeneities". For example, in the case of the usual Taylor expansions, we take for $A$ the set of natural numbers and $T_{\ell}$ contains the coefficients corresponding to the monomials of total degree $\ell$. In general, we only assume that the set $A$ is bounded from below and locally finite, and that each $T_{\alpha}$ is a real Banach space, although in many examples of interest these spaces will be finite-dimensional.

A crucial characteristic of Taylor expansions is that an expansion around any point $x_{0}$ can be re-expanded around any other point $x_{1}$, namely simply by making use of the identity

$$
\left(x-x_{0}\right)^{m}=\sum_{k+\ell=m}\left(\begin{array}{l}
m \\
k
\end{array}\right)\left(x_{1}-x_{0}\right)^{k} \cdot\left(x-x_{1}\right)^{\ell} .
$$

In the general case, we only assume that there are linear maps $\Gamma_{x y}$ transforming the coefficients of an expansion around $y$ into the coefficients for the same "polynomial", but this time expanded around $x$.

In view of the example of Taylor expansions, it is natural to impose that any such "reexpansion map" $\Gamma_{x y}$ has the property that if $\tau \in T_{\alpha}$, then $\Gamma_{x y} \tau-\tau \in$ $\bigoplus_{\beta<\alpha} T_{\beta}=: T_{<\alpha}$. In other words, when reexpanding a homogeneous monomial around a different point, the leading order coefficient remains the same, but lower order monomials may appear, just as is the case in (4.7). Furthermore, one should be able to compose reexpansions, since taking an expansion around $x$, reexpanding it around $y$ and then reexpanding the result around a third point $z$ should be the same as reexpanding the first expansion around $z$. In other words, it seems natural to impose the identity $\Gamma_{x y} \Gamma_{y z}=\Gamma_{x z}$. These considerations can be summarised in the following definition of an algebraic structure which we call a regularity structure:

Definition 4.2 Let $T=\bigoplus_{\alpha \in A} T_{\alpha}$ be a vector space graded by $A \subset \mathbf{R}$ (discrete bounded below) such that each $T_{\alpha}$ is a Banach space. Let furthermore $G$ be a group of continuous operators on $T$ such that, for every $\alpha \in A$, every $\Gamma \in G$, one has

$$
\tau \in T_{\alpha} \quad \Rightarrow \quad \Gamma \tau-\tau \in T_{<\alpha} .
$$

The pair $\mathscr{T}=(T, G)$ is called a regularity structure with model space $T$ and structure group $G$.

Remark 4.3 We say that an element $\tau \in T_{\alpha}$ is "homogeneous of degree $\alpha$ " and write $\operatorname{deg} \tau=\alpha$.

Such a regularity structure is a purely algebraic construct, but its purpose is to be endowed with some analytic "flesh": for each point $x$, we consider a linear map 
$\Pi_{x}: T \rightarrow \mathcal{S}^{\prime}$ (here $\mathcal{S}$ is the space of smooth test functions with compact support) with the property that, for every homogeneous $\tau$ of degree $\alpha$, the distribution $\Pi_{x} \tau$ satisfies an analytic bound that is homogeneous of degree $\alpha$ around $x$ :

$$
\left|\left(\Pi_{x} \tau\right)\left(\varphi_{x}^{\lambda}\right)\right| \lesssim \lambda^{\alpha}, \quad \forall \lambda \in(0,1],
$$

where $\varphi_{x}^{\lambda}$ denotes a test function $\varphi$ of size 1 , translated so that its support contains $x$, and then rescaled so that its integral doesn't change but the diameter of its support is of order $\lambda$. For a precise definition, see [Hai14, Def. 2.17].

The reexpansion property mentioned above then suggests that we should restrict our attentions to maps $\Pi$ as above with the additional property that one can find elements $\Gamma_{x y} \in G$ such that the identity

$$
\Pi_{x} \Gamma_{x y}=\Pi_{y},
$$

holds for any pair of points $(x, y)$. By scaling arguments and by analogy with (4.7) it is furthermore natural to impose that if $\tau \in T_{\alpha}$ and $\beta<\alpha$, then the component of $\Gamma_{x y} \tau$ in $T_{\beta}$ should be of size at most $\Theta\left(|x-y|^{\alpha-\beta}\right)$. We call such a pair $(\Pi, \Gamma)$ a "model" for the underlying regularity structure. The space $X$ of all models is endowed with a natural topology which turns it into a complete separable metric space, but not a linear space. In the analogy with usual Taylor polynomials, the regularity structure encodes the algebraic properties of Taylor monomials, while a model realises them as actual functions defined on some underlying Euclidean space.

Example 4.4 The usual Taylor polynomials are cast in this framework as follows. Take for $T$ the space of all polynomials $\sum_{k} a_{k} X^{k}$ in some abstract indeterminate $X$ (say there are d indeterminates so we interpret $k$ as a multiindex), with $T_{m}$ the subspace spanned by those $X^{k}$ with $|k|=m$. The group $G$ is then isomorphic to $\mathbf{R}^{d}$, acting on $T$ by $G_{h} X^{k}=(X-h)^{k}$ for $h \in \mathbf{R}^{d}$. The canonical model for this polynomial regularity structure is given by setting

$$
\left(\Pi_{x} X^{k}\right)(z)=(z-x)^{k}, \quad \Gamma_{x y}=G_{y-x} .
$$

It is then easy to check that (4.9) and (4.10) do indeed hold.

We can then consider functions $f: \mathbf{R}^{d} \rightarrow T$ which are interpreted as providing a "jet" $\Pi_{x} f(x)$ around every point $x$. Given a model, there are natural Hölder-type topology on the space of such functions which allow one to define analogues $\mathscr{D}^{\gamma}$ to the usual Hölder spaces $\mathscr{C}^{\gamma}$. The way these spaces are defined is completely analogous to the usual Hölder spaces in the sense that one would like $f$ to be "approximated by a polynomial up to order $\gamma$ ". In our context, a "polynomial" is naturally described by a function $p: \mathbf{R}^{d} \rightarrow T$ such that

$$
p(x)=\Gamma_{x y} p(y),
$$


for any two points $x$ and $y$. This function can be "reconstructed" to a bona fide distribution on $\mathbf{R}^{d}$ by setting $\mathscr{R} p=\Pi_{x} p(x)$. Note that, by (4.10) and (4.11), this is independent of the particular choice of $x$. (This is of course not to say that the distribution $\mathscr{R} p$ is constant!)

Given this discussion, it is natural to say that a function $f: \mathbf{R}^{d} \rightarrow T$ is in $\mathscr{D}^{\gamma}$ if (4.11) holds "up to order $\gamma$ ". More precisely, we impose that

$$
\left\|f(x)-\Gamma_{x y} f(y)\right\|_{\alpha} \lesssim|x-y|^{\gamma-\alpha}, \quad \alpha<\gamma,
$$

where $\|\cdot\|_{\alpha}$ denotes the norm of the component in $T_{\alpha}$ of some element of $T$. The reason for the exponent $\gamma-\alpha$ is that the component of $f$ in $T_{\alpha}$ should be thought of as representing a type of "derivative of order $\alpha$ ", again by analogy with usual Taylor expansions, so that (4.12) is analogous to the fact that if a function is of class $\mathscr{C}^{\gamma}$, then its derivative of order $\alpha$ is of class $\mathscr{C}^{\gamma-\alpha}$, provided of course that $\alpha<\gamma$. One fundamental result of the theory of regularity structures is the following, which is the analogue in this context of the "sewing lemma" of [Gubo4] and states that the operation $\mathscr{R}$ defined above on polynomials extends canonically to all of $\mathscr{D}^{\gamma}$ provided that $\gamma>0$.

Theorem 4.5 Given a regularity structure endowed with a model $Z=(\Pi, \Gamma)$, for any $f \in \mathscr{D}^{\gamma}$ with $\gamma>0$, there exists a unique distribution $\mathscr{R} f$ such that, for every $x \in \mathbf{R}^{d}$, the distribution $\Pi_{x} f(x)-\mathscr{R} f$ vanishes at order $\gamma$ in the vicinity of $x$.

Remark 4.6 We will sometimes write $\mathscr{R}(f, Z)$ instead to $\mathscr{R} f$ to emphasise the fact that this depends not only on the element $f \in \mathscr{D}^{\gamma}$, but also on the underlying model $Z \in X$.

Remark 4.7 In the case when $\Pi_{x} \tau$ is actually a continuous function for every $\tau \in T$ one has the explicit formula $(\mathscr{R} f)(x)=\left(\Pi_{x} f(x)\right)(x)$. In general, the right hand side of this expression makes of course no sense since $\Pi_{x} f(x)$ might be a distribution which cannot be evaluated pointwise.

Furthermore, the map $(Z, f) \mapsto \mathscr{R} f$ mapping a "model" $Z=(\Pi, \Gamma)$ as well as a "modelled distribution" $f \in \mathscr{D}^{\gamma}$ onto the Schwartz distribution $\mathscr{R} f$ is jointly continuous. (The precise continuity statement is somewhat subtle since the definition of the space $\mathscr{D}^{\gamma}$ is itself dependent of the model $Z$, so that the pairs $(Z, f)$ really take values in a type of topological vector bundle.) This theorem also justifies the terminology "model": the bound given in this theorem states that the distribution $\mathscr{R} f$ is modelled locally by $\Pi$ in the same way that a smooth function is modelled locally by polynomials.

The link to the discussion in Section 3.5 is now the following. The space $X$ is taken to be a suitable closed subset of the space of all models for a regularity 
structure that is canonically associated with a given class of semilinear parabolic stochastic PDEs. The map $\boldsymbol{M}_{A}$ is then built by mimicking the usual proof of well-posedness for parabolic PDEs, but in a suitable weighted version of the space $\mathscr{D}^{\gamma}$. This requires to build a whole calculus in these spaces in order to give a meaning to the various operations appearing there.

\subsection{Calculus for regularity structures}

Let us now show how one can construct the map $\varkappa_{A}: \mathcal{N} \times \mathcal{X} \rightarrow \mathcal{Y}$ alluded to earlier. Recall that $\mathcal{N}$ is a suitable space of right hand sides for the equation of interest and $X$ will be chosen as the space of models for a suitable regularity structure. The precise construction of the regularity structure in question is of course part of the question. The idea is to formulate an SPDE of the type (3.6) as a fixed point problem in some space $\mathscr{D}^{\gamma}$ and to then set

$$
\mathscr{M}_{A}(F, Z)=\mathscr{R}(\hat{M}(F, Z), Z),
$$

where $\hat{\mathscr{M}}: \mathcal{N} \times \mathscr{X} \rightarrow \mathscr{D}^{\gamma}$ is the solution to the fixed point problem and $\mathscr{R}$ is the reconstruction operator given by Theorem $4 \cdot 5$.

In order to construct a regularity structure adapted to an equation of the type (3.6), the idea is to start from the polynomial regularity structure endowed with its canonical model as described in Example 4.4 and to systematically enlarge it until it is sufficiently rich to support the operations required to formulate (3.6). Our first step is to add to the indeterminates $X_{i}$ additional symbols $\Xi_{i}$ on which the structure group acts trivially. The model $(\Pi, \Gamma)=\mathscr{L}(\xi)$ is then defined to be such that $\Pi_{x} \Xi_{i}=\xi_{i}$ for every $x$ and every $i$. The degree $\alpha_{i}=\operatorname{deg} \Xi_{i}$ of $\Xi_{i}$ is constrained by the regularity of the generalised random fields $\xi_{i}$ for which we want to build a solution theory in the sense that $\alpha_{i}$ should be sufficiently small so that the bound (4.9) holds for $\tau=\Xi_{i}$. In the case when $\xi_{i}$ is white noise for example, this imposes the constraint $\alpha_{i}<-\frac{D}{2}$, where $D$ is the scaling dimension of the underlying space(-time).

It is natural to also add symbols of the type $X^{k} \Xi_{i}$ to the collection of basis vectors of $T$ and to impose that $\operatorname{deg}\left(X^{k} \Xi_{i}\right)=\operatorname{deg} X^{k}+\operatorname{deg} \Xi_{i}=\alpha_{i}+|k|$. The natural action of $\mathbf{R}^{d}$ on these symbols is given by $G_{h}\left(X^{k} \Xi_{i}\right)=(X-h)^{k} \Xi_{i}$, and we naturally extend $\mathscr{L}(\xi)$ to it by setting $\left(\Pi_{x} X^{k} \Xi_{i}\right)(z)=(z-x)^{k} \xi_{i}(z)$. It is an easy exercise to verify that these definitions are compatible with (4.9) and (4.10).

Remark 4.8 In general, there is no reason to introduce symbols of the type $\Xi_{i} \Xi_{j}$ since products of noises do not naturally appear in 3.6 . Furthermore, in many situations there is no natural way of renormalising the pointwise product $\xi_{i} \xi_{j}$ in a meaningful way. As a consequence, our structure space $T$ will in general not be an algebra. Although our notation suggests that it is endowed with a product, this 
has a non-trivial domain of definition so that not any two vectors can be multiplied with each other.

At this stage, we note that we have enough structure to be able to use our theory to multiply the distributions $\xi_{i}$ with sufficiently regular functions $f \in \mathscr{C}^{\gamma}$. Indeed, given $f \in \mathscr{C}^{\gamma}$, we can canonically lift it to $F \in \mathscr{D}^{\gamma}$ by setting

$$
F(x)=\sum_{|k|<\gamma} \frac{f^{(k)}(x)}{k !} X^{k} .
$$

Since $F(x)$ lives in the polynomial "sector" of our regularity structure, we can multiply it by $\Xi_{i}$ and define $\hat{F}_{i}(x)=F(x) \Xi_{i}$. The condition $F \in \mathscr{D}^{\gamma}$ implies that $\hat{F}_{i} \in \mathscr{D}^{\gamma+\alpha_{i}}$ since

$$
\hat{F}_{i}(x)-\Gamma_{x y} \hat{F}_{i}(y)=\left(F_{i}(x)-\Gamma_{x y} F_{i}(y)\right) \Xi_{i},
$$

and multiplication by $\Xi_{i}$ changes the degree by $\alpha_{i}$, so that $\mathscr{R} \hat{F}_{i}$ is well-defined as soon as $\gamma$ is sufficiently large so that $\gamma+\alpha_{i}>0$. This is nothing but the well-known fact that the product $(f, \xi) \mapsto f \cdot \xi$ extends continuously to $\mathscr{C}^{\gamma} \times \mathscr{C}^{\alpha}$ if and only if $\gamma+\alpha>0$, see [BCD11], so it seems that we haven't really learned anything new yet.

However, one verifies that this construction generalises very easily. Indeed, consider any two subspaces $V_{1}, V_{2} \subset T$ that are invariant under the action of $G$, block-diagonal with respect to the decomposition $T=\bigoplus_{\alpha} T_{\alpha}$, and such that there exists a bilinear "product" $*: V_{1} \times V_{2} \rightarrow T$ with the additional property that, for every $\Gamma \in G$ and homogeneous $\tau_{i} \in V_{i}$, their product is homogeneous and one has

$$
\Gamma\left(\tau_{1} * \tau_{2}\right)=\left(\Gamma \tau_{1}\right) *\left(\Gamma \tau_{2}\right), \quad \operatorname{deg}\left(\tau_{1} * \tau_{2}\right)=\operatorname{deg} \tau_{1}+\operatorname{deg} \tau_{2} .
$$

We furthermore set $\beta_{i} \leq 0$ so that $V_{i} \subset \bigoplus_{\alpha \geq \beta_{i}} T_{\alpha}$. One then has the following general result, the proof of which is an elementary exercise.

Proposition 4.9 In the above context, given $F_{i} \in \mathscr{D}^{\gamma_{i}}$ with values in $V_{i}$ and $\gamma_{i}>0$, one has $F_{1} * F_{2} \in \mathscr{D}^{\gamma}$ with $\gamma=\left(\beta_{1}+\gamma_{2}\right) \wedge\left(\beta_{2}+\gamma_{1}\right)$.

Remark 4.10 In general, for $F_{i} \in T_{<\gamma_{i}}$ one does not have $F_{1} * F_{2} \in T_{<\gamma}$ but only $F_{1} * F_{2} \in T_{<\left(\gamma_{1}+\gamma_{2}\right)}$. In order to avoid this, one can define the product $*: \mathscr{D}^{\gamma_{1}} \times \mathscr{D}^{\gamma_{1}} \rightarrow \mathscr{D}^{\gamma}$ as

$$
\left(F_{1} * F_{2}\right)(z)=Q_{<\gamma}\left(F_{1}(z) * F_{2}(z)\right),
$$

where $Q_{<\gamma}$ is the projection onto $T_{<\gamma}$. It is a slightly lengthier exercise to verify that Proposition 4.9 still holds with this modified definition, so we will use this from now on. 
Note here that $F_{i}$ can be seen as having two distinct "regularities". Its "descriptive regularity" $\gamma_{i}>0$ measures how well $\mathscr{R} F_{i}$ can be described in terms of the underlying model, while its "scaling regularity" $\beta_{i} \leq 0$ measures the behaviour of $\mathscr{R} F_{i}$ around any given point in the sense that $\left(\mathscr{R} F_{i}\right)\left(\varphi_{x}^{\lambda}\right) \lesssim \lambda^{\beta_{i}}$. Note that the usual Hölder regularity of a continuous function is its descriptive regularity (with respect to the polynomial model), while its scaling regularity is always 0 . On the other hand, if we lift a distribution $\xi$ as above to a regularity structure via a suitable symbol $\Xi$, then the function $F(x) \mapsto \Xi$ satisfying $\mathscr{R} F=\xi$ has infinite descriptive regularity, while its scaling regularity coincides with the usual (negative) Hölder regularity of $\xi$. Proposition 4.9 can be interpreted as a rigorous formulation of the intuition that "multiplication by a distribution of regularity $-\alpha$ behaves like taking $\alpha$ derivatives".

Remark 4.11 At the algebraic level, it is not difficult to see that given $V_{1}$ and $V_{2}$ it is always possible to extend $T$ to some larger structure space $\tilde{T}$ while keeping $G$ fixed in such a way that on has a product $*: V_{1} \times V_{2} \rightarrow \tilde{T}$ and an action of $G$ on $\tilde{T}$ satisfying (4.15). It is however not clear in general how to extend a given model on $T$ to all of $T$. It was shown in [Hai14, Prop. 4.11] that it is actually always possible to do so, but while the construction given there is explicit, it relies on several arbitrary choices making this extension not canonical, except for homogeneous elements with degrees adding up to a strictly positive number. For example, in the situation of Remark 4.8 , that construction simply gives $\Pi_{x} \Xi_{i} \Xi_{j}=0$, which is not very satisfactory at all. In the particular case where the $\xi_{i}$ actually happen to be smooth, one would of course rather like to set $\Pi_{x} \Xi_{i} \Xi_{j}=\xi_{i} \xi_{j}$.

At this stage, our construction is already sufficiently rich to allow us to give a slightly cleaner formulation of the argument in Section 3.1. Indeed, ignoring for a moment the fact that one would like to impose an initial condition at time 0 , we reformulate (2.9) as an integral equation:

$$
\Phi=P \star\left(c \Phi-\Phi^{3}+\xi\right)=P \star\left(c \Phi-\Phi^{3}\right)+\Psi .
$$

If we now consider a regularity structure built from the polynomial one by adding symbols $\Psi^{i}$ for $i \leq 3$, we can then formulate this in a very natural way as a fixed point problem in $\mathscr{D}^{\gamma}$ :

$$
\Phi=\hat{\mathscr{L}}\left(P \star \mathscr{R}\left(c \Phi-\Phi^{3}\right)\right)+\Psi
$$

where we write $\hat{\mathscr{L}}$ for the map turning a continuous $\mathscr{C}^{\gamma}$ function into an element of $\mathscr{D}^{\gamma}$ as in (4.14). Under what conditions can we even find a space $\mathscr{D}^{\gamma}$ which is mapped into itself by the right hand side of (4.17)? By our definition, any solution $\Phi$ will be of the form $\Phi=\Psi+R$ for some remainder $R$ taking values 
in the polynomial sector, so that $\Phi^{3}$ does at least have a natural meaning in our regularity structure. Furthermore, if $\operatorname{deg} \Psi=-\alpha$, and $\Phi \in \mathscr{D}^{\gamma}$, we can apply Proposition 4.9 twice to find that $\Phi^{3} \in \mathscr{D}^{\gamma-2 \alpha}$, so that we certainly want to be able to take $\gamma>2 \alpha$ for the argument of $\mathscr{R}$ to be sufficiently regular.

On the other hand, it follows from (4.9) and Theorem 4.5 that one typically has $\mathscr{R} \Phi^{3} \in \mathscr{C}^{-3 \alpha}$ but no better so that, by the classical Schauder estimates, $\hat{\mathscr{L}} P \star$ $\mathscr{R} \Phi^{3} \in \mathscr{D}^{2-3 \alpha}$ but no better. These two conditions can be satisfied simultaneously provided that $2 \alpha<2-3 \alpha$, which restricts us to $\alpha<\frac{2}{5}$. However, if $\xi$ is space-time white noise, then it only belongs to $\mathscr{C}^{-\beta}$ for $\beta>\frac{d+2}{2}$, thus enforcing $\alpha>\frac{d-2}{2}$ which restricts us to dimensions $d<\frac{14}{5}$. In particular, this construction has no chance of covering the interesting case $d=3$.

The problem is that, in (4.17), we throw away all of the control that we have on $\Phi^{3}$ the moment we apply the reconstruction operator $\mathscr{R}$. Instead, we would like to construct an operator $\mathscr{P}$ which lifts the operation $P \star$ to the setting of modelled distributions in the sense that

$$
\mathscr{R} \mathscr{P} F=P \star \mathscr{R} F
$$

but that furthermore satisfies a type of Schauder estimate that increases both the descriptive and the scaling regularities by 2 (modulo the limitation that the scaling regularity can never become strictly positive).

In order to build such an operator, we need to be able to describe the local behaviour of $P \star \mathscr{R} F$ up to order $\gamma+2$, provided $F \in \mathscr{D}^{\gamma}$. Again, this requires our regularity structure to be sufficiently rich. It is natural to expect that if the local behaviour of $\mathscr{R} F$ involves $\Pi_{x} \tau$ for some $\tau$, then that of $P \star \mathscr{R} F$ will involve $P \star \Pi_{x} \tau$. Naïvely, this would suggest that we should consider regularity structures endowed with a map $\mathscr{I}$ such that

$$
\operatorname{deg} \mathscr{I} \tau=\operatorname{deg} \tau+2,
$$

and only consider models with the property that

$$
\Pi_{x} \mathscr{I} \tau=P \star \Pi_{x} \tau .
$$

Unfortunately, this is not compatible with the bound (4.9): even if $\operatorname{deg} \tau>-2$ for example, there is no reason whatsoever why $P \star \Pi_{x} \tau$ should vanish at $x$, which is imposed by (4.9). Instead, the idea is to force $\Pi_{x} \mathcal{F} \tau$ to vanish at the right order by considering models satisfying

$$
\Pi_{x} \mathscr{I} \tau=P \star \Pi_{x} \tau-\sum_{|k|<\operatorname{deg} \mathcal{I}_{\tau}} \frac{(\cdot-x)^{k}}{k !}\left(D^{k} P \star \Pi_{x} \tau\right)(x) .
$$

This immediately raises two questions: 
1. Since $D^{k} P \star \Pi_{x} \tau$ is a genuine distribution in general, what does it mean to evaluate it at $x$ ?

2. Is it always possible to construct a regularity structure and model with these properties and is this sufficient to construct the operator $\mathscr{P}$ ?

The first question can be addressed by realising that, by self-similarity, there exists a compactly supported test function $\varphi$ so that the heat kernel $P$ can be written as

$$
D^{k} P=\sum_{\log \lambda \in \mathbf{Z}} \lambda^{2-|k|}\left(D^{k} \varphi\right)_{0}^{\lambda},
$$

so that, at least formally, one has

$$
\left(D^{k} P \star \Pi_{x} \tau\right)(x)=\sum_{\log \lambda \in \mathbf{Z}} \lambda^{2-|k|}\left(\Pi_{x} \tau\right)\left(\left(D^{k} \varphi\right)_{x}^{\lambda}\right) .
$$

If we ignore the contributions coming from $\lambda>1$ (one can deal with these separately or replace the heat kernel by a truncated version), we see that this sum is guaranteed to converge precisely when $\alpha+2-|k|>0$, i.e. when $|k|<\operatorname{deg} \mathscr{I} \tau$.

Regarding the second question, it is shown in [Hai14, Sec. 4] that it does indeed have a positive answer in the following sense.

Theorem 4.12 Given a regularity structure $(T, G)$ extending the polynomial structure $(\bar{T}, \bar{G})$ and a subspace $V \subset T$ (block-diagonal and invariant under $G$ ), one can further extend $(T, G)$ to a regularity structure $(\tilde{T}, \tilde{G})$ on which there exists a map $\mathscr{I}: V \rightarrow \tilde{T}$ satisfying (4.19) and such that, for every $\Gamma \in \tilde{G}$ and $\tau \in V$, one has

$$
\Gamma \mathscr{I} \tau-\mathscr{I} \Gamma \tau \in \bar{T} .
$$

Furthermore, every model on $(T, G)$ can be canonically and continuously extended to a model on $(\tilde{T}, \tilde{G})$ satisfying (4.20). Finally, writing $\mathscr{D}^{\gamma}(V)$ for the space of $\mathscr{D}^{\gamma}$ functions with values in $V$, one can construct a continuous operator $\mathscr{P}: \mathscr{D}^{\gamma}(V) \rightarrow$ $D^{\gamma+2}$ such that 4.18 holds and such that furthermore

$$
(\mathscr{P} F)(x)-\mathscr{J}(F(x)) \in \bar{T} .
$$

\subsection{Construction of regularity structures for SPDEs}

We now have all the ingredients in place to construct the space $X$ and the map $\hat{M}$ appearing in (3.8) and (4.13). To construct the regularity structure, start with symbols $1, X_{i}$ (one for every space-time coordinate) and $\Xi_{j}$ (one for every driving noise) with degrees given as above. From these, we then build a collection $\mathscr{W}$ of symbols by inductively postulating that

$$
\tau_{1}, \tau_{2} \in \mathscr{W} \quad \Rightarrow \quad \tau_{1} \tau_{2} \in \mathscr{W}, \quad \operatorname{deg} \tau_{1} \tau_{2}=\operatorname{deg} \tau_{1}+\operatorname{deg} \tau_{2}
$$




$$
\tau \in \mathscr{W} \quad \Rightarrow \quad \mathscr{I}(\tau) \in \mathscr{W}, \quad \operatorname{deg} \mathscr{I}(\tau)=2+\operatorname{deg} \tau .
$$

We put on $\mathscr{W}$ the equivalence relation $\sim$ generated by $1 \tau \sim \tau, \tau_{1} \tau_{2} \sim \tau_{2} \tau_{1}$, $\left(\tau_{1} \tau_{2}\right) \tau_{3} \sim \tau_{1}\left(\tau_{2} \tau_{3}\right)$, and by also postulating that $\tau_{1} \sim \tau_{2}$ implies both $\mathscr{S}\left(\tau_{1}\right) \sim$ $\mathscr{I}\left(\tau_{2}\right)$ and $\tau_{1} \tau \sim \tau_{2} \tau$. We then write $\tilde{\mathscr{W}}=\mathscr{W} / \sim$ so that $\tilde{\mathscr{W}}$ is a graded commutative monoid endowed with a map $\mathscr{I}$.

The set $\tilde{\mathscr{V}}$ is much too large to be a good set of basis vectors for a regularity structure since the corresponding set of degrees is typically neither discrete nor bounded from below. One therefore selects the smallest subset $\mathscr{F} \subset \tilde{\mathscr{W}}$ which is sufficiently large so that it is possible to formulate the SPDE under consideration as a fixed point problem in the regularity structure built by taking $T=\langle\mathscr{F}\rangle$ and for $G$ the group of all linear operators on $T$ such that $\Gamma X_{i}-X_{i} \in\langle\mathbf{1}\rangle, \Gamma \Xi_{i}=\Xi_{i}$, and satisfying (4.8), (4.15) and (4.21). (The set $\mathscr{F}$ needs to be chosen sufficiently large so that any linear map $\Gamma$ on $\langle\mathscr{W}\rangle$ satisfying all of the above properties maps $\langle\mathscr{F}\rangle$ to itself.)

An equation is said to be locally subcritical if the corresponding set $\mathscr{F}$ is such that, for every $\gamma \in \mathbf{R}$, there are only finitely many elements $\tau \in \mathscr{F}$ with $\operatorname{deg} \tau<\gamma$, which is a prerequisite for $(T, G)$ as above to fulfil the axioms of a regularity structure. Let us go back to our running example of the $\Phi^{4}$ model. In this case, ignoring again the effect of initial conditions, we would like to rewrite 4.16 as a fixed point problem in some $\mathscr{D}^{\gamma}$ space as follows:

$$
\Phi=\mathscr{P}\left(c \Phi-\Phi^{3}+\Xi\right) .
$$

As a consequence of Theorem 4.12 , this equation is of the form

$$
\Phi=\mathscr{I}\left(c \Phi-\Phi^{3}+\Xi\right)+(\ldots),
$$

where (...) denotes terms belonging to $\bar{T}$. Let us write $\mathscr{F}_{0}$ for the collection of basis vectors required to describe $\Phi$ and $\mathscr{F}_{0}$ for those required to describe $\Phi^{3}$. We then certainly want $X^{k} \in \mathscr{F}_{0}$ for all $k$ as well as $\mathscr{I}(\Xi) \in \mathscr{F}_{0}$. Furthermore, whenever $\tau \in \mathscr{F}_{1}$, we want to have $\mathscr{F}(\tau) \in \mathscr{F}_{0}$. Regarding $\mathscr{F}_{1}$, whenever $\tau_{1}, \tau_{2}, \tau_{3} \in \mathscr{F}_{0}$, we want to have $\tau_{1} \tau_{2} \tau_{3} \in \mathscr{F}_{1}$, so that $\Phi^{3} \in\left\langle\mathscr{F}_{1}\right\rangle$ for all $\Phi \in\left\langle\mathscr{F}_{0}\right\rangle$.

To simplify notations, let us introduce graphical notations where we denote $\Xi$ by a dot and $\mathscr{I}$ by a line, and where multiplication is denoted by joining graphs by their roots. For example, we write $\mathscr{I}(\Xi)=1, \mathscr{I}(\Xi)^{2}=v, \mathscr{I}\left(\mathscr{I}(\Xi)^{2}\right) \mathscr{I}(\Xi)^{2}=\mho$, etc. With this notation, we then have

$$
\begin{aligned}
& \left.\mathscr{F}_{0}=\{\uparrow,\rangle, \varphi, \varphi, \ldots\right\},
\end{aligned}
$$

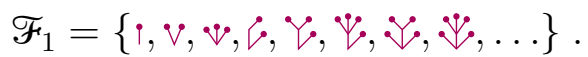

If we set $\operatorname{deg} \Xi=-\alpha$, then we have $\operatorname{deg} \uparrow=2-\alpha, \operatorname{deg} \vartheta=6-3 \alpha, \operatorname{deg} \mho=10-$ $4 \alpha$, etc. One can verify that the condition for subcriticality is that $\operatorname{deg} \vee>\operatorname{deg} \Xi$, 
namely that $\Xi$ is the term of lowest degree appearing on the right hand side of (4.22). This imposes that $\alpha>-3$ so that, since space-time white noise has scaling exponent $-\frac{d+2}{2}$ the critical dimension is indeed 4, which is consistent with the heuristic presented in Section 3.4.

It should be clear that this procedure is rather robust and allows us to associate a regularity structure to any locally subcritical stochastic PDE. Furthermore, the regularity structures built with this procedure are endowed with a canonical lift $\mathscr{L}: \mathscr{B}_{\infty} \rightarrow \mathscr{X}$ (recall that $X$ is the space of models for our regularity structure) by setting

$$
\Pi_{x} \Xi_{i}=\xi_{i}, \quad\left(\Pi_{x} X_{i}\right)(z)=z_{i}-x_{i},
$$

and then extending this to all of $\mathscr{F}$ by imposing that

$$
\Pi_{x}(\tau \bar{\tau})=\left(\Pi_{x} \tau\right) \cdot\left(\Pi_{x} \bar{\tau}\right)
$$

as well as (4.20). There is also a natural (essentially unique) way of choosing $\Gamma_{x y} \in G$ such that (4.10) holds and it is possible to verify that this choice of ( $\Pi, \Gamma$ ) satisfies the bound (4.9) as well as the required analytical bound on $\Gamma_{x y}$, provided that $\xi$ is smooth.

\section{Renormalisation}

Let us quickly summarise the situation so far. Given a class of stochastic PDEs, we have just seen how to build a regularity structure in which we can use the calculus developed in Section $4 \cdot 3$ in order to reformulate the equation as a fixed point problem in some space $\mathscr{D}^{\gamma}$. In general, this equation may not have a solution but, if one considers periodic (in space) situations, it was shown in [Hai14] that it is possible to define weighted versions of the spaces $\mathscr{D}^{\gamma}$ in which the right hand side of (4.22) (or the analogous mild formulation of the SPDE under consideration, taking furthermore the effect of the initial condition into account) defines a contraction when restricted to short enough time intervals, so that it admits a solution $\hat{\mathscr{M}}$ which depends continuously on the underlying model $Z \in X$.

Via the reconstruction operator $\mathscr{R}, \hat{M}$ then defines a solution map $\mathcal{M}_{A}$ by (4.13). This construction implements the strategy outlined in Section 3.5: the lift $\mathscr{L}$ is given by the canonical lift as above with the left inverse $\pi$ given trivially by $(\pi(\Pi, \Gamma))_{i}=\Pi_{0} \Xi_{i}$. This is indeed surjective on $\mathscr{B}$ provided that we choose $\operatorname{deg} \Xi_{i}=\alpha_{i}-\kappa$, since the bound (4.9) is equivalent to the $\mathscr{C}^{\alpha}$ norm cases where $\Gamma_{x y}$ acts trivially on $\tau$ (as it does on $\Xi_{i}$ by construction). Furthermore, the identity (4.24) combined with Remark 4.7 implies that, for smooth models, one has

$$
\mathscr{R}(F \cdot G)=\mathscr{R} F \cdot \mathscr{R} G,
$$

which in turn, when combined with 4.18), shows that the first identity in 3.8 holds. 


\subsection{Alternative representation of models}

The only remaining problem is that, as we have already seen in the calculations of Section 4.1 , the canonical lift $\xi \mapsto \mathscr{L}(\xi)$ does not extend continuously to all $\xi \in \mathscr{B}$. Instead, we would like to perform a suitable renormalisation procedure as described in Section 3.5. Before we do this, we note that the canonical lift $\mathscr{L}$ can alternatively be described in a slightly different way. Given a smooth $\xi \in \mathscr{B}_{\infty}$, we obtain a representation $\Pi$ of our regularity structure by setting similarly to above $(\Pi \mathbf{1})(x)=1,\left(\Pi X_{i}\right)(x)=x_{i},\left(\Pi \Xi_{i}\right)(x)=\xi_{i}(x)$, and then extending this inductively to all of $T$ by

$$
\Pi(\tau \bar{\tau})=(\Pi \tau) \cdot(\Pi \bar{\tau}), \quad \Pi \mathscr{I}(\tau)=P \star \Pi \tau .
$$

This is very similar to the construction of $\mathscr{L}(\xi)$ given above, except that we dropped the polynomial correction term in (4.20). One then has the following alternative characterisation of $\mathscr{L}(\xi)$ which is relatively straightforward to show by induction.

Proposition 5.1 Given $\xi \in \mathscr{B}_{\infty}$, define $\Pi$ as above. Then, for every $x$, there exists an essentially unique $F_{x} \in G$ such that $\left(D^{k} \Pi F_{x} \tau\right)(x)=0$ for every $\tau \in \mathscr{F}$ and every $|k|<\operatorname{deg} \tau$. Furthermore, writing $\mathscr{L}(\xi)=(\Pi, \Gamma)$, one has

$$
\Pi_{x}=\Pi F_{x} \quad \text { and } \quad \Gamma_{x y}=F_{x}^{-1} F_{y} .
$$

Remark 5.2 By "essentially unique", we mean that $F_{x}$ is uniquely determined by $\Pi$ for generic choices of $\Pi$ (and therefore $\xi$ ). In special cases as when $\xi=0$ this is of course not the case. See [BHZ16, Prop. 6.3] for a more precise formulation. The condition $\left(D^{k} \Pi F_{x} \tau\right)(x)=0$ for $|k|<\operatorname{deg} \tau$ combined with the smoothness of $\Pi$ immediately implies (4.9).

Remark 5.3 For arbitrary $\Pi: T \rightarrow \mathscr{C}^{\infty}$, we can still find a (generically unique) $F_{x}^{\Pi} \in G$ such that the condition $\left(D^{k} \Pi F_{x} \tau\right)(x)=0$ for $|k|<\operatorname{deg} \tau$ holds for all $\tau$ of the form $\tau=\mathscr{I}(\sigma)$ for some $\sigma \in \mathscr{F}$. This does however not imply in general that this bound (and therefore (4.9)) holds for arbitrary $\tau$ !

Write now $x_{\infty}$ for the collection of all smooth maps

$$
\Pi: T \rightarrow \mathscr{C}^{\infty} \quad \text { such that } \quad \Pi \mathscr{I}(\tau)=P \star \Pi \tau
$$

and such that one can find a map $x \mapsto F_{x} \in G$ such that (5.3) defines a model $(\Pi, \Gamma) \in \mathcal{X}$. While Proposition 5.1 states that the canonical representation $\Pi$ built from $\xi \in \mathscr{B}_{\infty}$ does belong to $X_{\infty}$, it is not the case in general that $X_{\infty}$ contains all maps $\Pi$ satisfying (5.4).

Since every smooth model is built from a map $\Pi$ as in (5.3) (take for example $\Pi=\Pi_{0}$ and $F_{x}=\Gamma_{0, x}$ ), we henceforth describe models via such maps. Since the 
maps $\Pi$ are linear, it is natural in view of Section $3 \cdot 5$ to look for a group $\Re$ of linear maps $M: T \rightarrow T$ acting on $\mathcal{X}_{\infty}$ on the right by $\Pi \mapsto \Pi \circ M$. We furthermore want our renormalisation procedure to keep the meanings of the symbols $1, X$ and $\Xi$ intact and to preserve the second identity in (5.2). We therefore look for maps $M: T \rightarrow T$ satisfying the following properties

1. One has $M \mathbf{1}=\mathbf{1}$ and $M \Xi_{i}=\Xi_{i}$ for all $i$.

2. One has $M X_{i} \tau=X_{i} M \tau$ and $M \mathscr{I}(\tau)=\mathscr{I}(M \tau)$ for all $\tau \in T$.

3. For every $\Pi \in X_{\infty}$, one has $\Pi \circ M \in X_{\infty}$.

The first two properties are straightforward to verify, but it is much less clear $a$ priori how the last one can be checked. A group $\mathfrak{R}$ of linear maps satisfying all of these properties was exhibited in [BHZ16, Thm 6.28], (it is called $\mathscr{G}_{-}$in that article) where it was also shown that $\mathfrak{R}$ is sufficiently large to be able to find elements $g^{\xi} \in \mathfrak{R}$ such that $(\overline{3.9})$ holds. (Here, $X_{\infty}$ plays the role of the linear space $\hat{\mathscr{X}}$ alluded to in Remark 3.4 and $\hat{\mathscr{L}}$ is given by the canonical lift $\xi \mapsto \Pi$ described above.)

\subsection{Description of the renormalisation procedure}

We do not give a full description of the group $\mathfrak{R}$ here, but rather a slightly simplified one corresponding to a subgroup of the group $\mathscr{G}_{-}$described in [BHZ16]. The idea is that each element $M_{g} \in \mathfrak{R}$ is described by a map $g: \mathscr{F}_{-} \rightarrow \mathbf{R}$, where $\mathscr{F}_{-} \subset \mathscr{F}$ denotes the subset of $\mathscr{F}$ consisting of symbols with strictly negative degree and not containing any of the symbols $X_{i}$. As in Section 4.4, we identify elements of $\mathscr{F}$ with trees $\mathfrak{T}=(V, E)$ whose nodes $V$ are endowed with labels describing the index $i$ of the corresponding term $\Xi_{i}$. If the symbol in question also contains powers of $X$, then we encode these as additional labels on the nodes of the tree, so that nodes are indexed by elements in $\mathbf{N}^{d} \times\{0, \ldots, m\}$ where a noise index of 0 denotes the absence of noise. For example, $X_{2} \mathscr{I}\left(\Xi_{1}\right) \mathscr{I}\left(\Xi_{2} X_{1}\right)$ is identified with the tree $v$, where the two leaves have labels $((), 1)$ and $((1), 2)$ respectively, while the root has label $((2), 0)$. (We denote multiindices by unordered tuples.)

Given such a labelled tree $\mathfrak{T}$, we write $\overline{\mathfrak{T}} \subset \mathfrak{T}$ for a subgraph consisting of a collection of edges $\bar{E} \subset E$, as well as all the vertices $\bar{V} \subset V$ incident to $\bar{E}$. Given $\overline{\mathfrak{T}} \subset \mathfrak{T}$, we denote by $\iota(\overline{\mathfrak{T}})$ the element of the free algebra $\left\langle\left\langle\mathscr{F}_{-}\right\rangle\right\rangle$generated by $\mathscr{F}_{-}$ by identifying each connected component of $\overline{\mathfrak{T}}$ with an element of $\mathscr{F}_{-}$(ignoring the values of the $X$-component of the labels of the nodes of $\overline{\mathfrak{T}}$ ) and multiplying them in $\left\langle\left\langle\mathscr{F}_{-}\right\rangle\right\rangle$. If one of the connected components of $\overline{\mathfrak{T}}$ happens to have positive degree, we set $\iota(\overline{\mathfrak{T}})=0$. We naturally map the empty subgraph to the unit of $\left\langle\left\langle\mathscr{F}_{-}\right\rangle\right\rangle$.

Given $\overline{\mathfrak{T}} \subset \mathfrak{T}$, we also denote by $\mathfrak{T} / \overline{\mathfrak{T}}$ the new element of $\mathscr{F}$ obtained by contracting each connected component $A$ of $\overline{\mathfrak{T}}$ to a single vertex. The label of this vertex is given by $(k, 0)$, where $k$ is the sum of all the $X$-labels of vertices of $A$. 
For example, we have

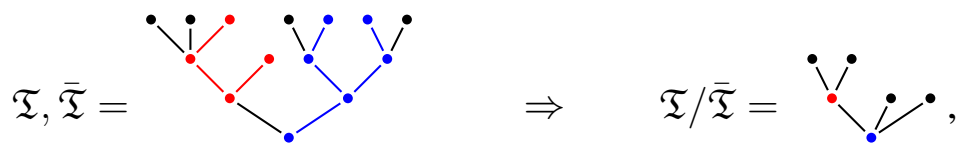

where $\overline{\mathfrak{T}}$ is the coloured subgraph of $\mathfrak{T}$, with the two connected components drawn in blue and red respectively. If the labels of the red vertices in $\mathfrak{T}$ are labelled by $\left(k_{i}, j_{i}\right)$, then the label of the single red vertex in $\mathfrak{T} / \overline{\mathfrak{T}}$ is $(k, 0)$ with $k=\sum_{i} k_{i}$.

Given $g: \mathscr{F}_{-} \rightarrow \mathbf{R}$, we then also write $g$ for its unique extension to a character of $\left\langle\left\langle\mathscr{F}_{-}\right\rangle\right\rangle$. The above definitions then allow us to define a map $M_{g}: T \rightarrow T$ by

$$
M_{g} \mathfrak{T}=\sum_{\overline{\mathfrak{T}} \subset \mathfrak{T}} g(\iota(\overline{\mathfrak{T}})) \mathfrak{T} / \overline{\mathfrak{T}}
$$

It is not very difficult to verify that one has $M_{f} \circ M_{g}=M_{f \circ g}$, where the composition rule between maps $f, g: \mathscr{F}_{-} \rightarrow \mathbf{R}$ is given by

$$
(f \circ g)(\mathfrak{T})=\sum_{\overline{\mathfrak{T}} \subset \mathfrak{T}} g(\iota(\overline{\mathfrak{T}})) f(\mathfrak{T} / \overline{\mathfrak{T}})
$$

again with the conventions that $g$ is extended to all of $\left\langle\left\langle\mathscr{F}_{-}\right\rangle\right\rangle$as above and that $f(\mathfrak{T} / \overline{\mathfrak{T}})=0$ if its argument happens to be of positive degree. Thanks to the fact that, as soon as $\overline{\mathfrak{T}} \neq \varnothing, \mathfrak{T} / \overline{\mathfrak{T}}$ has strictly less edges than $\mathfrak{T}$, each operator $M_{f}$ differs from the identity by a (locally) nilpotent operator, so that the Neumann series for its inverse converges. One can show that this inverse is of the form $M_{f^{-1}}$ for some character $f^{-1}$, and that there exists a linear map $\mathscr{A}:\left\langle\mathscr{F}_{-}\right\rangle \rightarrow\left\langle\mathscr{F}_{-}\right\rangle$such that $f^{-1}=f \circ \mathscr{A}$. (As a matter of fact, this endows $\left\langle\mathscr{F}_{-}\right\rangle$with a Hopf algebra structure and (5.5) turns $T$ into a left comodule for $\left\langle\mathscr{F}_{-}\right\rangle$.)

Remark 5.4 Recall that the group $\mathfrak{R}$ constructed here is in general a proper subgroup of the full group $\mathscr{G}_{-}$described in [BHZ16] which is required for the renormalisation procedure to work in general. The full group is constructed similarly to above, but without the restriction that elements of $\mathscr{F}_{-}$have vanishing $X$-labels. In this case however, formula (5.5) has to be replaced by a more complicated version which includes a suitable action on those labels. Since this would only distract from the gist of the argument, we refrain from presenting this here.

\subsection{Action on the space of models}

Denoting by $\Re$ the group of all characters of $\left\langle\left\langle\mathscr{F}_{-}\right\rangle\right\rangle$endowed with the composition rule [5.6), we obtained in [BHZ16, Thm 6.28] the following result. 
Theorem 5.5 The formula $(f, \Pi) \mapsto \Pi \circ M_{f}$ yields a (right) group action of $\mathfrak{R}$ onto $X_{\infty}$ which extends to a continuous group action on $X$.

The purpose of this section is to give an idea how the proof of this result works. Assume for a moment that the map $(g, F) \mapsto F^{g}=M_{g}^{-1} F M_{g}$ yields a group action of $\mathfrak{R}$ onto $G$ by a group automorphisms such that, for every $\Pi: T \rightarrow \mathscr{C}^{\infty}$ and every $g \in \mathfrak{R}$, writing $F_{x}^{\Pi}$ for the element of $G$ described in Remark $5 \cdot 3$, one has

$$
M_{g} F_{x}^{\Pi M_{g}}=F_{x}^{\Pi} M_{g},
$$

or equivalently $\left(F_{x}^{\Pi}\right)^{g}=F_{x}^{\Pi M_{g}}$.

If this were the case then, writing $\Pi^{g}=\Pi M_{g}$, we would have the identity

$$
\Pi_{x}^{g} \stackrel{\text { def }}{=} \Pi^{g} F_{x}^{\Pi^{g}}=\Pi M_{g}\left(F_{x}^{\Pi}\right)^{g}=\Pi F_{x}^{\Pi} M_{g}=\Pi_{x} M_{g} .
$$

Since, by the definition (5.5), $M_{g} \tau-\tau \in T_{>\operatorname{deg} \tau}$, it immediately follows that $\Pi \in X_{\infty}$ implies $\Pi^{g} \in X_{\infty}$. Unfortunately, it turns out that the map $(g, F) \mapsto$ $F^{g}=M_{g}^{-1} F M_{g}$ does not have these properties in general.

The way around this is to construct a larger regularity structure $\left(T^{\mathrm{ex}}, G^{\mathrm{ex}}\right)$ which extends $(T, G)$ in the sense that $T \hookrightarrow T^{\mathrm{ex}}, G^{\mathrm{ex}} \rightarrow G$, and the action of $G^{\mathrm{ex}}$ on $T^{\mathrm{ex}}$ leaves $T$ invariant and coincides with that of $G$ there. This extension furthermore comes with a natural projection $\pi: T^{\mathrm{ex}} \rightarrow T$ as well as with maps $\Pi^{\mathrm{ex}}: F_{x}^{\mathrm{ex}} \in G^{\mathrm{ex}}$ such that the collection of maps $\left(\Pi_{x}^{\mathrm{ex}}, \Gamma_{x y}^{\mathrm{ex}}\right)$ given by

$$
\Pi_{x}^{\mathrm{ex}}=\Pi^{\mathrm{ex}} F_{x}^{\mathrm{ex}}, \quad \Gamma_{x y}^{\mathrm{ex}}=\left(F_{x}^{\mathrm{ex}}\right)^{-1} F_{y}^{\mathrm{ex}},
$$

form a model for $\left(T^{\mathrm{ex}}, G^{\mathrm{ex}}\right)$ in the case where $\Pi^{\mathrm{ex}}$ is of the form $\Pi^{\mathrm{ex}}=\Pi \pi$ for $\Pi$ the canonical lift of a smooth noise $\xi$. The first step of the proof is then to show that the map $\iota: \Pi \mapsto \Pi \pi$ extends to a continuous injection of $X_{\infty}$ into $X_{\infty}^{\mathrm{ex}}$. The second step is to show that $\mathfrak{R}$ also has a natural action $g \mapsto M_{g}^{\mathrm{ex}}$ on $T^{\mathrm{ex}}$ which is compatible with that on $T$ in the sense that

$$
\pi M_{g}^{\mathrm{ex}}=M_{g} \pi
$$

In particular, this implies that $\iota\left(\Pi M_{g}\right)=\iota(\Pi) M_{g}^{\mathrm{ex}}$. The third step then consists in verifying that for the extended regularity structure, it is indeed the case that (5.7) holds, thus yielding a right action of $\mathfrak{R}$ onto $X_{\infty}^{\mathrm{ex}}$. Finally, one concludes the argument by showing that this action leaves $X_{\infty}$ (viewed as a subset of $X_{\infty}^{\text {ex }}$ via $\iota$ ) invariant.

It remains to describe the extended regularity structure. The idea is to consider a set $\tilde{\mathscr{W}}^{\text {ex }}$ consisting of all labelled trees like $\tilde{\mathscr{W}}$, but this time with labels in $\mathbf{N}^{d} \times\{0, \ldots, m\} \times \mathbf{R}_{-}$, i.e. we add an additional "extended" $\mathbf{R}_{-}$-valued label to each node. We write $\pi: \tilde{\mathscr{W}}^{\text {ex }} \rightarrow \tilde{\mathscr{W}}$ for the map that simply forgets the extended 
label. Writing $\beta_{u}$ for the extended label of the node $u$, we also define the degree of $\mathfrak{T} \in \tilde{\mathscr{W}}^{\text {ex }}$ by setting

$$
\operatorname{deg} \mathfrak{T}=\operatorname{deg} \pi \mathfrak{T}+\sum_{u \in V} \beta_{u} .
$$

Given such a labelled tree $\mathfrak{T} \in \tilde{\mathscr{N}}$ as well as a subgraph $\overline{\mathfrak{T}} \subset \mathfrak{T}$ as above, we then define $\iota(\overline{\mathfrak{T}}) \in\left\langle\left\langle\tilde{\mathscr{W}}^{\text {ex }}\right\rangle\right\rangle$ just as above, but we do keep the values of the extended labels on the extracted subgraph. We also define $\mathfrak{T} / / \overline{\mathfrak{T}}$ as in Section 5.2 , but with the extended label of any new vertex $u$ in $\mathfrak{T} / / \overline{\mathfrak{T}}$ corresponding to the contraction of a connected component $A \subset \mathfrak{T}$ of $\overline{\mathfrak{T}}$ given by $\beta_{u}=\operatorname{deg} \iota(A)$. We then define $\mathscr{F}^{\text {ex }} \subset \tilde{\mathscr{W}}^{\text {ex }}$ as the set of all $\mathfrak{T}$ for which there exists $\hat{\mathfrak{T}} \in \mathscr{F}$ and $\overline{\mathfrak{T}} \subset \hat{\mathfrak{T}}$ such that $\mathfrak{T}=\hat{\mathfrak{T}} / / \overline{\mathfrak{T}}$.

The structure space for the extended regularity structure is then defined as $T^{\mathrm{ex}}=\left\langle\mathscr{F}^{\mathrm{ex}}\right\rangle$. This space still admits a natural (partial) product obtained by joining trees by their roots as above and setting the extended label at the root of the resulting tree to be the sum of the extended labels at the roots of the two factors. Similarly, it admits a map $\mathscr{I}$ in the same way as above, setting the extended label of the newly created root to 0 . We can furthermore introduce maps $L_{\alpha}$ that act on a labelled tree $\mathfrak{T}$ by adding $\alpha$ to the extended label of the root. We then define the group $G^{\mathrm{ex}}$ of all linear operators $\Gamma$ on $T^{\mathrm{ex}}$ such that $\Gamma X_{i}-X_{i} \in\langle\mathbf{1}\rangle, \Gamma \Xi_{i}=\Xi_{i}$, and satisfying (4.8), (4.15) and (4.21). We furthermore restrict $G^{\text {ex }}$ to elements such that $\Gamma L_{\alpha}(\mathfrak{T})=L_{\alpha}(\Gamma \mathfrak{T})$ whenever $\mathfrak{T} \in \mathscr{F}^{\mathrm{ex}}$ is such that one also has $L_{\alpha}(\mathfrak{T}) \in \mathscr{F}^{\mathrm{ex}}$. It is clear by construction that any such map does act as some element of $G$ on the subspace $T \subset T^{\mathrm{ex}}$, so that the resulting regularity structure does indeed extend $(T, G)$.

This allows us to build an "extended" renormalisation group $\mathfrak{R}^{\text {ex }}$ exactly as above, but keeping track of the extended labels. In other words, we define $\mathscr{F}_{-}^{\mathrm{ex}} \subset$ $\mathscr{F}^{\mathrm{ex}}$ as the subset of all elements of $\mathscr{F}^{\mathrm{ex}}$ of strictly negative degree and with vanishing $X$-labels (but possibly non-vanishing extended labels). Elements of $\mathfrak{R}^{\text {ex }}$ are then described by maps $g: \mathscr{F}_{-}^{\mathrm{ex}} \rightarrow \mathbf{R}$ and their action on $T^{\mathrm{ex}}$ is given as above by

$$
M_{g} \mathfrak{T}=\sum_{\overline{\mathfrak{T}} \subset \mathfrak{T}} g(\iota(\overline{\mathfrak{T}})) \mathfrak{T} / / \overline{\mathfrak{T}} .
$$

One can verify that the map $g \mapsto g^{\text {ex }}=g \circ \pi$ allows to view $\mathfrak{R}$ as a subgroup of $\mathfrak{R}^{\mathrm{ex}}$, thus yielding an action of $\mathfrak{R}$ on $T^{\mathrm{ex}}$ which does indeed satisfy (15.9).

We claim that this action now does satisfy the identity (5.7). The main reason for this is that, by the definition of the operation // and the degree on $\mathscr{F}^{\mathrm{ex}}$, all terms appearing in the right hand side of (5.10) have the same degree. As a consequence, one can easily verify that if we define $\Pi_{x}^{g}=\Pi_{x} M_{g}^{\text {ex }}$ as in (5.8), then it does satisfy (4.20). Since the latter determines the characters $F_{x}^{\Pi}$, one can use this to go backwards and show that (5.7) does indeed hold. 


\subsection{Renormalised equations}

In this last section, we show how one constructs the 'dual' right action of $\Re$ onto the space of equations in such a way that the identity (3.11) holds. Formalising the space $\mathcal{N}$ of "possible right hand sides" is rather heavy notationally, so we restrict ourselves again to the specific example of the $\Phi^{4}$ model, although the argument is quite general.

We consider the case of $d=3$, so that we can choose $\alpha=\frac{5}{2}+\kappa$ for some sufficiently small $\kappa>0$. In particular, one has $\operatorname{deg} \uparrow=-\frac{1}{2}-\kappa$, so that Proposition $4 \cdot 9$ tells us that we should look for solutions to (4.22) in $\mathscr{D}^{\gamma}$ for some $\gamma>1+2 \kappa$. Choosing $\gamma=1+3 \kappa$ and $\kappa$ small enough, we then note that as a consequence of (4.23), every solution to the fixed point problem (4.22) is of the form

$$
\Phi=\uparrow+\varphi \mathbf{1}-\ddot{\psi}-3 \varphi \hat{Y}+\langle\nabla \varphi, X\rangle
$$

for some continuous functions $\varphi$ and $\nabla \varphi$.

Remark 5.6 By Remark 4.10, the identity (5.11) is exact, not just some approximate identity "up to higher order terms". The continuous functions $\varphi$ and $\nabla \varphi$ however are unknown and to be determined by the fixed point problem. Note also that $\nabla \varphi$ is not the gradient of $\varphi$, but can be interpreted as a kind of "renormalised gradient". In fact, it follows from (4.12) and (4.20) that for every $\Phi \in \mathscr{D}^{\gamma}$ of the form $(\overline{5.11})$, one has the identity

$\left(\nabla_{i} \varphi\right)(z)=\lim _{h \rightarrow 0} h^{-1}\left(\varphi\left(z+h e_{i}\right)-\varphi(z)-\left(\Pi_{z} \psi\right)\left(z+h e_{i}\right)-3 \varphi(z)\left(\Pi_{z} Y\right)\left(z+h e_{i}\right)\right)$,

with $e_{i}$ the $i$ th unit vector in $\mathbf{R}^{3}$. The mere fact that there even exist functions such that this limit is finite for every $z$ is not obvious!

As a consequence of $(5.11)$ and Remark 4.10 , one has the identity

$$
\Phi^{3}=\ddot{\vee}+3 \varphi \vee+3 \varphi^{2} \uparrow+\varphi^{3} \mathbf{1}-6 \varphi \vartheta-3 \ddot{\vee}-9 \varphi \dot{\vartheta}+3\langle\nabla \varphi, X\rangle \vee .
$$

Again, this is true whatever the underlying model $\Pi$, as long as it is admissible in the sense that the second identity in (5.2) holds, whether it is obtained from the canonical lift of a smooth noise, or not. Of course the actual function / distribution $\mathscr{R} \Phi$ represented by this identity depends very much on $\Pi$, first because the reconstruction operator $\mathscr{R}$ depends on it and second because the function $\varphi$ (and therefore also $\nabla \varphi$ ) depends on it.

Consider now the particular case when $\Pi$ is the canonical lift of some smooth noise $\xi$. In this case, it follows from (5.1) that $\mathscr{R}\left(\Phi^{3}\right)=(\mathscr{R} \Phi)^{3}$. Let now $g$ be an element of $\mathfrak{R}$, write $\hat{\Pi}=\Pi \circ M_{g}$, and write $\hat{\mathscr{R}}$ for the corresponding reconstruction operator. Making use of the extended model described in the previous subsection, 
combined with Remark 4.7 , we can write this renormalised reconstruction operator as

$$
(\hat{\mathscr{R}} U)(z)=\left(\Pi_{z}^{\mathrm{ex}} M_{g}^{\mathrm{ex}} U(z)\right)(z)=\left(\mathscr{R} M_{g}^{\mathrm{ex}} U\right)(z) .
$$

At this point, we choose $g: \mathscr{F}_{-} \rightarrow \mathbf{R}$ as

$$
g(\vee)=-c_{1}, \quad g(\vee)=-c_{2}
$$

and $g(\tau)=0$ otherwise. It then follows from $(\overline{5 \cdot 5})$ that one has for example

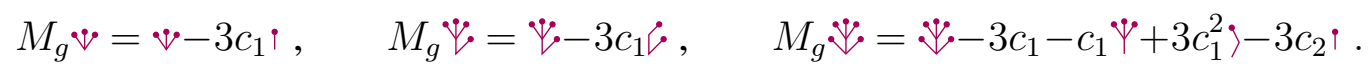

The action of $M_{g}^{\text {ex }}$ is essentially the same, but with extended decorations with value $-1-2 \kappa$ added on those vertices obtained by contracting an instance of $v$.

Inserting this into $(\overline{5.12})$, it follows that one has the identities

$$
M_{g}^{\mathrm{ex}} \Phi \sim \Phi, \quad M_{g}^{\mathrm{ex}} \Phi^{3} \sim \Phi^{3}-\left(3 c_{1}-9 c_{2}\right) \Phi,
$$

where we write $\tau \sim \bar{\tau}$ if $\left(\Pi_{z}^{\mathrm{ex}} \tau\right)(z)=\left(\Pi_{z}^{\mathrm{ex}} \bar{\tau}\right)(z)$. We conclude that in this particular example one has

$$
\hat{\mathscr{R}}\left(\Phi^{3}\right)=(\hat{\mathscr{R}} \Phi)^{3}-\left(3 c_{1}-9 c_{2}\right) \hat{\mathscr{R}} \Phi .
$$

Take now as our space $\mathcal{N}$ of nonlinearities all nonlinearities of the type $F_{c}(\Phi)=$ $c \Phi-\Phi^{3}$ for $c \in \mathbf{R}$. It then follows from (5.14) that, at least for the subgroup of $\mathfrak{R}$ given by elements of the type (5.13) (which as a group is simply $\left(\mathbf{R}^{2},+\right)$ ), one does indeed have the announced identity $(3.11)$ with

$$
F_{c} g=F_{c+3 c_{1}-9 c_{2}} \text {. }
$$

Remark 5.7 In the case $c_{2}=0$ (which is the case in dimension 2), we see that we recover the calculation performed in (3.4).

Remark 5.8 The observant reader may have noticed that we have

$$
g\left(\Phi^{3}\right)=-3 c_{1} \varphi+9 c_{2} \varphi,
$$

which appears coincidentally to be "essentially the same" as the counterterm $-3 c_{1} \hat{\mathscr{R}} \Phi+9 c_{2} \hat{\mathscr{R}} \Phi$ appearing in (5.14). This is actually not a coincidence but holds in very wide generality, see [BCCH17]. (Compare Eq. 2.15 with Def. 3.15 and Lemma 4.5, noting that the symmetry factor $S(\tau)$ is generated by the scalar product appearing in the left hand side of Def. 3.15.) This gives us a very simple way of deriving the renormalisation procedure for any given class of stochastic PDEs. 


\section{References}

[BC14] A. Borodin and I. Conwin. Macdonald processes. Probab. Theory Related Fields 158, no. 1-2, (2014), 225-400. http://dx.doi.org/10.1007/ s00440-013-0482-3.

[BCCH17] Y. Bruned, A. Chandra, I. Chevyrev, and M. Hairer. Renormalizing SPDEs in regularity structures. ArXiv e-prints (2017). http://arxiv.org/ abs/1711.10239.

[BCD11] H. Bahouri, J.-Y. Chemin, and R. Danchin. Fourier analysis and nonlinear partial differential equations, vol. 343 of Grundlehren der Mathematischen Wissenschaften [Fundamental Principles of Mathematical Sciences]. Springer, Heidelberg, 2011.

[BFS83] D. C. Brydges, J. Fröhlich, and A. D. Sokal. The random-walk representation of classical spin systems and correlation inequalities. Communications in Mathematical Physics 91, no. 1, (1983), 117-139. http://dx.doi.org/ 10.1007/BF01206055.

[BG97] L. Bertini and G. Giacomin. Stochastic Burgers and KPZ equations from particle systems. Comm. Math. Phys. 183, no. 3, (1997), 571-607. http:// dx.doi.org/10.1007/s002200050044.

[BHZ16] Y. Bruned, M. Hairer, and L. Zambotti. Algebraic renormalisation of regularity structures. ArXiv e-prints (2016). http://arxiv.org/abs/1610. 08468.

[Boug6] J. Bourgain. Invariant measures for the 2D-defocusing nonlinear Schrödinger equation. Comm. Math. Phys. 176, no. 2, (1996), 421-445.

[CGN15] F. Camia, C. Garban, and C. M. Newman. Planar Ising magnetization field I. Uniqueness of the critical scaling limit. Ann. Probab. 43, no. 2, (2015), 528-571. http://arxiv.org/abs/1205.6610. http://dx.doi.org/10. 1214/13-AOP881.

[CGN16] F. Camia, C. Garban, and C. M. Newman. Planar Ising magnetization field II. Properties of the critical and near-critical scaling limits. Ann. Inst. Henri Poincaré Probab. Stat. 52, no. 1, (2016), 146-161. http://arxiv.org/ abs/1307. 3926. http://dx.doi.org/10.1214/14-AIHP643.

[CH16] A. Chandra and M. Hairer. An analytic BPHZ theorem for regularity structures. ArXiv e-prints (2016). http://arxiv.org/abs/1612.08138.

[CQR 15] I. CoRwin, J. Quastel, and D. RemeniK. Renormalization fixed point of the KPZ universality class. J. Stat. Phys. 16o, no. 4, (2015), 815-834. http:// dx.doi.org/10.1007/s10955-015-1243-8.

[CS12] D. ChelkaK and S. Smirnov. Universality in the $2 \mathrm{D}$ Ising model and conformal invariance of fermionic observables. Invent. Math. 189, no. 3, 
(2012), 515-580. http://arxiv.org/abs/0910.2045. http://dx.doi. org/10.1007/s00222-011-0371-2.

[CS16] I. CoRwin and H. Shen. Open ASEP in the Weakly Asymmetric Regime. ArXiv e-prints (2016). http://arxiv. org/abs/1610.04931.

[DMIPP84] A. De Masi, N. Ianiro, A. Pellegrinotti, and E. Presutti. A survey of the hydrodynamical behavior of many-particle systems. In Nonequilibrium phenomena, II, Stud. Statist. Mech., XI, 123-294. North-Holland, Amsterdam, 1984 .

[Don51] M. D. DonsKer. An invariance principle for certain probability limit theorems. Mem. Amer. Math. Soc., No. 6, (1951), 12.

[DPDo2] G. Da Prato and A. Debussche. Two-dimensional Navier-Stokes equations driven by a space-time white noise. J. Funct. Anal. 196, no. 1, (2002), 180-210. http://dx.doi.org/10.1006/jfan.2002.3919.

[DPDo3] G. Da Prato and A. Debussche. Strong solutions to the stochastic quantization equations. Ann. Probab. 31, no. 4, (2003), 1900-1916. http://dx. doi.org/10.1214/aop/1068646370.

$\left[\mathrm{ESPP}^{+}{ }_{12}\right.$ ] S. El-Showk, M. F. Paulos, D. Poland, S. Rychkov, D. Simmons-Duffin, and A. Vichi. Solving the $3 \mathrm{~d}$ Ising model with the conformal bootstrap. Phys. Rev. D 86, (2012), 025022. http://arxiv.org/abs/1203.6064. http:// dx.doi.org/10.1103/PhysRevD. 86.025022.

$\left[\right.$ ESPP $\left.^{+}{ }_{14}\right]$ S. El-Showk, M. F. Paulos, D. Poland, S. Rychkov, D. Simmons-Duffin, and A. VICHI. Solving the $3 \mathrm{~d}$ Ising model with the conformal bootstrap II. c-minimization and precise critical exponents. J. Stat. Phys. 157, no. 4-5, (2014), 869-914. http://arxiv.org/abs/1403.4545. http://dx.doi. org/10.1007/s10955-014-1042-7.

[FH14] P. K. Friz and M. HaIRER. A course on rough paths. Universitext. Springer, Cham, 2014. With an introduction to regularity structures. http://dx. doi . org/10.1007/978-3-319-08332-2.

[FQ15] T. FunAKI and J. QuAstel. KPZ equation, its renormalization and invariant measures. Stoch. Partial Differ. Equ. Anal. Comput. 3, no. 2, (2015), 159220. http://arxiv.org/abs/1407.7310. http://dx.doi.org/10.1007/ s40072-015-0046-x.

[FV 10] P. K. Friz and N. B. VICTOIR. Multidimensional stochastic processes as rough paths, vol. 120 of Cambridge Studies in Advanced Mathematics. Cambridge University Press, Cambridge, 2010. Theory and applications. http://dx. doi.org/10.1017/CB09780511845079.

[GH18] M. Gerencsér and M. Hairer. Singular spdes in domains with boundaries. Probab. Theory Related Fields (2018). http://arxiv.org/abs/ 1702.06522. http://dx.doi.org/10.1007/s00440-018-0841-1. 
[GJ87] J. Glimm and A. JAFFe. Quantum physics. Springer-Verlag, New York, second ed., 1987. A functional integral point of view. http://dx.doi.org/ 10. 1007/978-1-4612-4728-9.

[GJ14] P. c. Gonçalves and M. JARA. Nonlinear fluctuations of weakly asymmetric interacting particle systems. Arch. Ration. Mech. Anal. 212, no. 2, (2014), 597-644. http://arxiv.org/abs/1309.5120. http://dx.doi.org/10. 1007/s00205-013-0693-x.

[GP16] M. Gubinelli and N. Perkowski. The Hairer-Quastel universality result in equilibrium. ArXiv e-prints (2016). http://arxiv.org/abs/1602.02428.

[GP18] M. Gubinelli and N. Perkowski. Energy solutions of KPZ are unique. $J$. Amer. Math. Soc. 31, no. 2, (2018), 427-471. http://arxiv.org/abs/ 1508.07764 .

[Gubo4] M. GubinelLi. Controlling rough paths. J. Funct. Anal. 216, no. 1, (2004), 86-140. http://arxiv.org/abs/math/0306433. http://dx.doi.org/ $10.1016 / j . j f a .2004 .01 .002$.

[Hai14] M. HaIrer. A theory of regularity structures. Invent. Math. 198, no. 2, (2014), 269-504. http://arxiv.org/abs/1303.5113. http://dx.doi. org/10.1007/s00222-014-0505-4.

[Hai17] M. HAIRER. An analyst's take on the BPHZ theorem. ArXiv e-prints (2017). http://arxiv.org/abs/1704.08634.

[HQ15] M. Hairer and J. QuAstel. A class of growth models rescaling to KPZ. ArXiv e-prints (2015). http://arxiv.org/abs/1512.07845.

[HRW 12] M. Hairer, M. D. Ryser, and H. Weber. Triviality of the 2D stochastic AllenCahn equation. Electron. J. Probab. 17, (2012), no. 39, 14. http://arxiv. org/abs/1201.3089. http://dx.doi.org/10.1214/EJP.v17-1731.

[HX18a] M. HaIRER and W. Xu. Large-scale behavior of three-dimensional continuous phase coexistence models. Comm. Pure Appl. Math. 71, no. 4, (2018), 688-746. http://arxiv.org/abs/1601.05138. http://dx.doi.org/10. 1002/cpa. 21738.

[HX18b] M. Hairer and W. Xu. Large-scale limit of interface fluctuation models. ArXiv e-prints (2018). http://arxiv.org/abs/1802.08192.

[Johoo] K. Johansson. Shape fluctuations and random matrices. Comm. Math. Phys. 209, no. 2, (2000), 437-476. http://dx.doi.org/10.1007/ s002200050027.

[Kes8o] H. Kesten. The critical probability of bond percolation on the square lattice equals $\frac{1}{2}$. Comm. Math. Phys. 74, no. 1, (1980), 41-59. http://dx.doi. org/10.1007/BF01197577. 
[KPZ86] M. Kardar, G. Parisi, and Y.-C. Zhang. Dynamic scaling of growing interfaces. Phys. Rev. Lett. 56, no. 9, (1986), 889-892. http://dx.doi. org/10.1103/PhysRevLett.56.889.

[LCLo7] T. J. Lyons, M. Caruana, and T. LÉvy. Differential equations driven by rough paths, vol. 1908 of Lecture Notes in Mathematics. Springer, Berlin, 2007. Lectures from the 34th Summer School on Probability Theory held in Saint-Flour, July 6-24, 2004, With an introduction concerning the Summer School by Jean Picard.

[Lig85] T. M. LigGETT. Interacting particle systems, vol. 276 of Grundlehren der Mathematischen Wissenschaften [Fundamental Principles of Mathematical Sciences]. Springer-Verlag, New York, 1985. http://dx.doi.org/10. 1007/978-1-4613-8542-4.

[LQ02] T. LyOns and Z. Qian. System control and rough paths. Oxford Mathematical Monographs. Oxford University Press, Oxford, 2002. Oxford Science Publications. http://dx.doi.org/10.1093/acprof:oso/ 9780198506485.001 .0001 .

[Lyo91] T. Lyons. On the nonexistence of path integrals. Proc. Roy. Soc. London Ser. A 432, no. 1885, (1991), 281-290. http://dx.doi.org/10.1098/rspa. 1991.0017.

[Lyo98] T. J. Lyons. Differential equations driven by rough signals. Rev. Mat. Iberoamericana 14, no. 2, (1998), 215-310. http://dx.doi.org/10.4171/ $\mathrm{RMI} / 240$.

[MQR17] K. Matetski, J. Quastel, and D. Remenik. The KPZ fixed point. ArXiv e-prints (2017). http://arxiv.org/abs/1701.00018.

[MW 17] J.-C. Mourrat and H. Weber. Convergence of the two-dimensional dynamic Ising-Kac model to $\Phi_{2}^{4}$. Comm. Pure Appl. Math. 7o, no. 4, (2017), 717-812. http://arxiv.org/abs/1410.1179. http://dx.doi.org/10.1002/cpa. 21655.

[Ne166] E. Nelson. A quartic interaction in two dimensions. In Mathematical Theory of Elementary Particles (Proc. Conf., Dedham, Mass., 1965), 69-73. M.I.T. Press, Cambridge, Mass., 1966.

[QS15] J. Quastel and H. Spohn. The one-dimensional KPZ equation and its universality class. J. Stat. Phys. 16o, no. 4, (2015), 965-984. http://arxiv.org/ abs/1503.06185, http://dx.doi.org/10.1007/s10955-015-1250-9.

[Rav92] K. Ravishankar. Fluctuations from the hydrodynamical limit for the symmetric simple exclusion in $\mathbf{Z}^{d}$. Stochastic Process. Appl. 42, no. 1, (1992), 31-37. http://dx.doi.org/10.1016/0304-4149(92)90024-K.

[SS11] O. Schramm and S. Smirnov. On the scaling limits of planar percolation. Ann. Probab. 39, no. 5, (2011), 1768-1814. With an appendix by Christophe 
Garban. http://arxiv.org/abs/1101.5820. http://dx.doi.org/10. 1214/11-AOP659.

[Tsio4] B. Tsirelson. Scaling limit, noise, stability. In Lectures on probability theory and statistics, vol. 1840 of Lecture Notes in Math., 1-106. Springer, Berlin, 2004. http://dx.doi.org/10.1007/978-3-540-39982-7_1.

[TWo8] C. A. TRACY and H. Widom. Integral formulas for the asymmetric simple exclusion process. Comm. Math. Phys. 279, no. 3, (2008), 815-844. http:// dx.doi.org/10.1007/s00220-008-0443-3.

[Wei6o] S. Weinberg. High-energy behavior in quantum field-theory. Phys. Rev. (2) 118, (1960), 838-849. http://dx.doi.org/10.1103/PhysRev.118.838. 